\title{
Odd-parity spin-loop-current order mediated by transverse spin fluctuations in cuprates and related electron systems
}

\author{
Hiroshi Kontani $\odot$, Youichi Yamakawa, Rina Tazai, and Seiichiro Onari \\ Department of Physics, Nagoya Furo-cho, Nagoya 464-8602, Japan
}

(Received 24 February 2020; revised 23 August 2020; accepted 22 December 2020; published 10 February 2021)

\begin{abstract}
Unconventional symmetry-breaking phenomena due to nontrivial order parameters attract increasing attention in strongly correlated electron systems. Here, we predict theoretically the occurrence of nanoscale spontaneous spin current, called the spin-loop-current (sLC) order, as a promising origin of the pseudogap and electronic nematicity in cuprates. We reveal that the sLC is driven by the odd-parity electron-hole condensations that are mediated by transverse spin fluctuations around the pseudogap temperature $T^{*}$. At the same temperature, odd-parity magnon pair condensation occurs. The sLC order is "hidden" in that neither internal magnetic field nor charge-density modulation is induced, whereas the predicted SLC with finite wave-number naturally gives the Fermi arc structure. In addition, the fluctuations of sLC order work as attractive pairing interaction between adjacent hot spots, which enlarges the $d$-wave superconducting transition temperature $T_{\mathrm{c}}$. The sLC state will be a key ingredient in understanding the pseudogap, electronic nematicity, as well as superconductivity in cuprates and other strongly correlated metals.
\end{abstract}

DOI: 10.1103/PhysRevResearch.3.013127

\section{INTRODUCTION}

Various unconventional symmetry-breaking phenomena, such as violations of rotational and parity symmetries, have been discovered in many strongly correlated metals recently. This fact strongly indicates the emergence of exotic density-wave orders, which are totally different from usual spin/charge-density waves. Various exotic symmetrybreaking phenomena, such as violations of rotational and parity symmetries, are the central issues in cuprate high- $T_{\mathrm{c}}$ superconductors. However, their microscopic mechanisms still remain as unsolved issues. Figure 1(a) shows a schematic phase diagram of cuprate superconductors. Below $T_{\mathrm{CDW}} \sim$ $200 \mathrm{~K}$, a stripe charge-channel density wave emerges at finite wave vector $\boldsymbol{q} \approx(\pi / 2,0)$ in many compounds [1-4], which produces the Fermi arc structure and causes a reduction in the density of states (DOS). However, it cannot be the origin of the pseudogap temperature $T^{*}$ since $T^{*}>T_{\mathrm{CDw}}$. Short quasiparticle lifetime due to spin or charge fluctuations could reduce the DOS [5-7].

Recently, much experimental evidence for the phase transition at $T^{*}$ has been accumulated [8-13]. Various fascinating order parameters have been proposed and actively investigated, such as the charge-density wave (CDW) or bond order (BO) [14-23], the pair-density wave [24,25], and the chargeloop-current (cLC) order [26-29]. At present, the symmetries of the hidden order in the pseudogap phase are not yet confirmed experimentally. Thus, it is necessary to study various

Published by the American Physical Society under the terms of the Creative Commons Attribution 4.0 International license. Further distribution of this work must maintain attribution to the author(s) and the published article's title, journal citation, and DOI. possibilities without prejudice based on advanced many-body theories [15-29].

Let us discuss the symmetry breaking in the correlated hopping between sites $i$ and $j: t_{i, j} \rightarrow t_{i, j}+\delta t_{i, j}$, where $\delta t_{i, j}$ $\left(=\delta t_{j, i}\right)^{*}$ is the order parameter. Then, the $\mathrm{BO}$ is given by a real and even-parity $\delta t_{i, j}[14,15,17-23]$. A spin-fluctuation mechanism [22,23] predicts the ferro $(\boldsymbol{q}=\mathbf{0}) d$-wave BO state at $T^{*}$ and stripe $[\boldsymbol{q} \approx(\pi / 2,0)] \mathrm{BO}$ at $T_{\mathrm{CDW}}$. The former order explains the experimental nematic transition $[10,13]$. However, simple translational symmetry preserving ferro-BO does not explain the pseudogap formation. Also, the cLC order is given by a pure imaginary and odd-parity $\delta t_{i, j}$ [26-29]. Both order parameters have been actively investigated.

In contrast, spin current flows if the pure imaginary order parameter is odd under space and spin inversions: $\delta t_{i, j}^{\sigma}=$ $-\delta t_{j, i}^{\sigma}=-\delta t_{i, j}^{-\sigma}$ as shown in Fig. 1(b) [29-34]. Here, $\sigma= \pm 1$ represents the spin of the electron. Figures 1(c) and 1(d) depict the spin-loop-current (sLC) order at the wave vectors $\boldsymbol{q}_{\mathrm{sLC}}=$ $(\delta, \delta)$ and that at $\boldsymbol{q}_{\mathrm{sLC}}=(\delta, 0)$ with $\delta=\pi / 2$, respectively. The sLC is a hidden order in the sense that no internal magnetic field appears, and charge-density modulation is quite small. Nonetheless, the sLC is very attracting since the pseudogap and Fermi surface (FS) reconstruction are induced by band folding if $\boldsymbol{q}_{\mathrm{sLC}} \neq \mathbf{0}$.

In this paper, we discover the emergence of "hidden symmetry breaking" accompanied by finite spin current at $\boldsymbol{q}_{\mathrm{sLC}} \approx(\pi / 2, \pi / 2)$. This sLC order originates from the spinflipping magnon-exchange process, called the AslamazovLarkin (AL) process [16,35-37]. The sLC order is hidden in that neither internal magnetic field nor charge-density modulation is induced, while the band folding by these sLC orders produces the Fermi arc structure and pseudogap in the DOS $[9,38]$. The derived transition temperature $T_{\mathrm{sLC}}$ is higher than that of the stripe $\mathrm{BO}$, and comparable to that of the ferro-BO. The sLC order will be responsible for the pseudogap and 


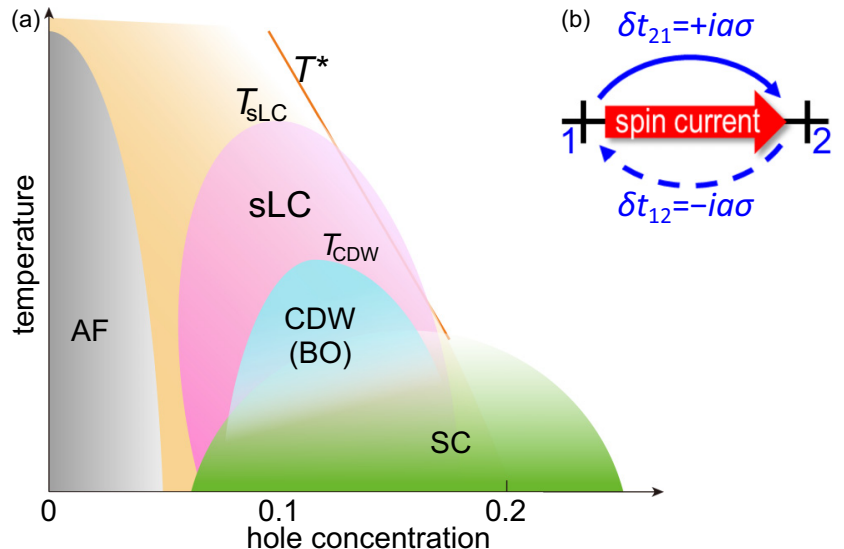

(c) diagonal SLC
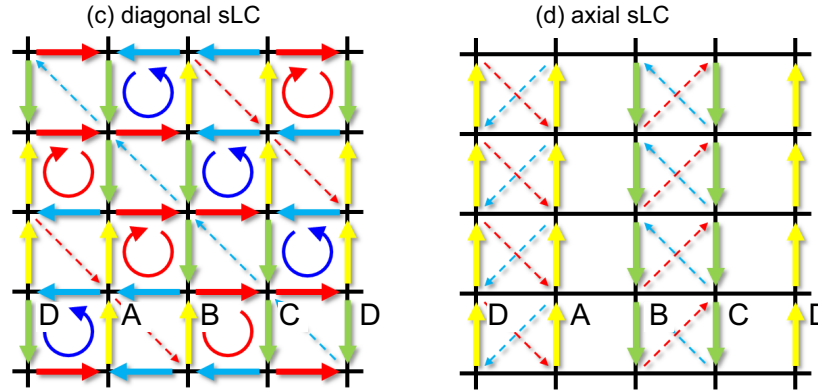

FIG. 1. (a) Possible phase diagram of hole-doped cuprate superconductors. The sLC phase is obtained by the present study. (b) Current of $\operatorname{spin}(\sigma= \pm 1)$ from site 1 to site $2 . \sigma= \pm 1$ is the spin of the electron. (c),(d) Schematic pictures of the diagonal sLC at $\boldsymbol{q}_{\mathrm{sLC}}=(\pi / 2, \pi / 2)$ and the axial $\operatorname{sLC}$ at $\boldsymbol{q}_{\mathrm{sLC}}=(\pi / 2,0)$, respectively.

electronic nematicity not only in cuprates, but also in iridates and $f$-electron systems [32-34,39].

The emergence of the sLC has been discussed in various electronic systems [29-33]. From the microscopic viewpoint, however, the mechanism of the sLC is highly nontrivial, since the realization condition of the sLC order is very severe in the extended $U-V-J$ Hubbard model within the mean-field theory [30]. In addition, only the case $\boldsymbol{q}_{\mathrm{sLC}}=(\pi, \pi)$ was analyzed in previous works. The present study can explain the sLC order based on a simple Hubbard model with on-site $U$, without assuming the wave vector $\boldsymbol{q}_{\mathrm{sLC}}$.

\section{SPIN-FLUCTUATION-DRIVEN UNCONVENTIONAL ORDERS}

\section{A. Model Hamiltonian}

Here, we analyze the single-orbital square-lattice Hubbard model

$$
H=\sum_{k, \sigma} \epsilon_{k} c_{k \sigma}^{\dagger} c_{k \sigma}+U \sum_{i} n_{i \uparrow} n_{i \downarrow} .
$$

We denote the hopping integrals $\left(t_{1}, t_{2}, t_{3}\right)=$ $(-1,1 / 6,-1 / 5)$, where $t_{l}$ is the $l$ th nearest hopping integral $[40,41]$. Hereafter, we set the unit of energy as $\left|t_{1}\right|=1$, which corresponds to $\sim 4000 \mathrm{~K}$ in cuprates, and fix the temperature $T=0.05(\sim 200 \mathrm{~K})$. The FS at filling $n=0.85$ is given in Fig. 2(a). The spin
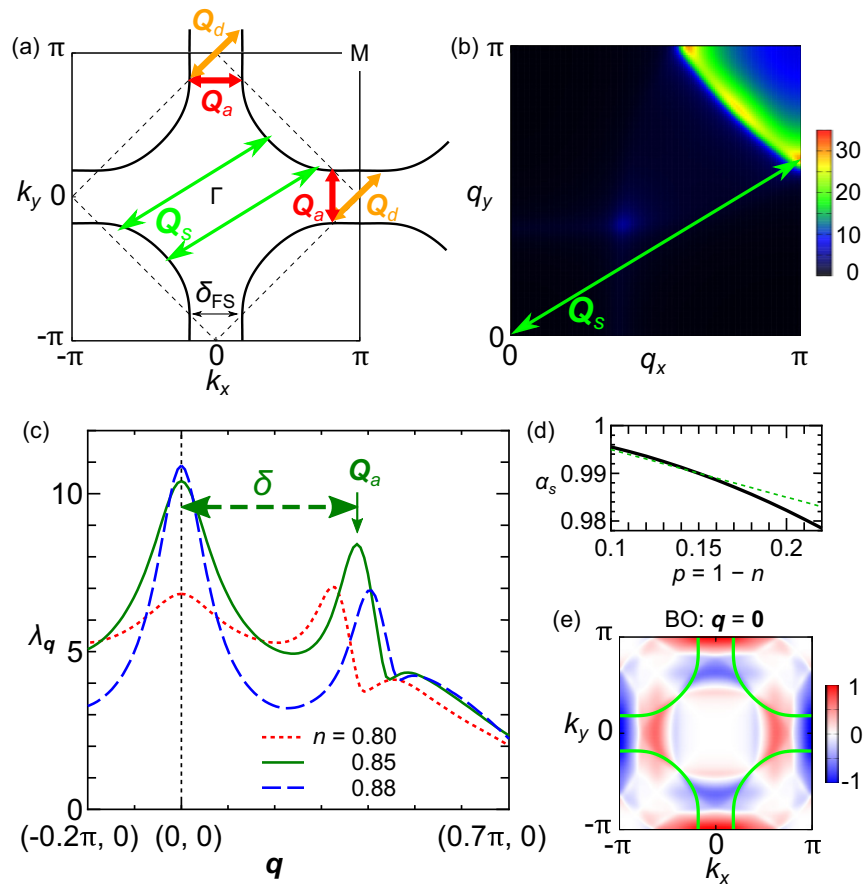

FIG. 2. (a) The FS of the present model at $n=0.85 . \boldsymbol{Q}_{s}$ are the major nesting vectors. $\boldsymbol{Q}_{\mathrm{d}}=(\delta, \delta)$ and $\boldsymbol{Q}_{\mathrm{a}}=(\delta, 0)\left(\delta \approx \delta_{\mathrm{FS}}\right)$ are minor nesting vectors. They correspond to the sLC order wavelength in the present theory. (b) $\chi^{s}(\boldsymbol{q})$ given by the RPA. It shows the incommensurate peak at $\boldsymbol{q}=\boldsymbol{Q}_{s}$. (c) Obtained eigenvalue $\lambda_{q}$ for the BO at $n=0.80-0.88$. They have peaks at $\boldsymbol{q}=\mathbf{0}$ and $\boldsymbol{q}=\boldsymbol{Q}_{\mathrm{a}}$. (d) Relations $\alpha_{S}=1-0.444 p^{2}$ (full line) and $\alpha_{S}=1.01-0.2 p$ (broken line). (e) Obtained $d$-wave form factor $f_{q}(\boldsymbol{k})$ for $\boldsymbol{q}=\mathbf{0}$ together with the FS.

susceptibility in the random-phase-approximation (RPA) is $\chi^{s}(q)=\chi^{0}(q) /\left[1-U \chi^{0}(q)\right]$, where $\chi^{0}(q)$ is the irreducible susceptibility without $U$ and $q \equiv\left(\boldsymbol{q}, \omega_{l}\right)$. The spin Stoner factor is defined as $\alpha_{S} \equiv \max _{q}\left\{U \chi^{0}(q)\right\}=U \chi^{0}\left(\boldsymbol{Q}_{s}, 0\right)$. Figure 2(b) shows the obtained $\chi^{s}(q)$ at $\alpha_{S}=0.99$ $(U=3.27)$. Here, $\chi^{s}\left(\boldsymbol{Q}_{s}, 0\right) \sim 30\left[1 / t_{1}\right] \sim 80\left[\mu_{\mathrm{B}}^{2} / \mathrm{eV}\right]$, which is still smaller than $\operatorname{Im} \chi^{s}\left(\boldsymbol{Q}_{s}, E=31 \mathrm{meV}\right) \sim 200\left[\mu_{\mathrm{B}}^{2} / \mathrm{eV}\right]$ at $T \sim 200 \mathrm{~K}$ in $60 \mathrm{~K} \mathrm{YBCO} \mathrm{[42].} \mathrm{Thus,} \alpha_{S}>0.99$ in real compounds. Owing to the Mermin-Wagner theorem, the relation $\alpha_{S} \lesssim 1$ is naturally satisfied for $U \gg 3.3$ without any fine tuning of $U$ by considering the spin-fluctuation-induced self-energy self-consistently [40].

\section{B. Introduction of singlet and triplet DW equations}

From now on, we investigate possible exotic density-wave (DW) states for both charge and spin channels with general wave vector $(\boldsymbol{q})$, which is generally expressed as [30]

$$
\begin{aligned}
D_{\boldsymbol{q}}^{\sigma \rho}(\boldsymbol{k}) & =\left\langle c_{\boldsymbol{k}_{-}, \sigma}^{\dagger} c_{\boldsymbol{k}_{+}, \rho}\right\rangle-\left\langle c_{\boldsymbol{k}_{-}, \sigma}^{\dagger} c_{\boldsymbol{k}_{+}, \rho}\right\rangle_{0} \\
& =f_{\boldsymbol{q}}(\boldsymbol{k}) \delta_{\sigma, \rho}+\boldsymbol{g}_{\boldsymbol{q}}(\boldsymbol{k}) \cdot \boldsymbol{\sigma}_{\sigma, \rho},
\end{aligned}
$$

where $\boldsymbol{k}_{ \pm} \equiv \boldsymbol{k} \pm \boldsymbol{q} / 2$, and $d_{\boldsymbol{q}}^{c}(\boldsymbol{k})\left[\boldsymbol{d}_{\boldsymbol{q}}^{s}(\boldsymbol{k})\right]$ is the charge (spin) channel order parameter. It induces the symmetry breaking in the self-energy:

$$
\Delta \Sigma_{\boldsymbol{q}}^{\sigma \rho}(\boldsymbol{k})=f_{\boldsymbol{q}}(\boldsymbol{k}) \delta_{\sigma, \rho}+\boldsymbol{g}_{\boldsymbol{q}}(\boldsymbol{k}) \cdot \boldsymbol{\sigma}_{\sigma, \rho},
$$


which we call the form factors in this paper. Below, we assume $\boldsymbol{g}_{\boldsymbol{q}}(\boldsymbol{k})=g_{\boldsymbol{q}}(\boldsymbol{k}) \boldsymbol{e}_{z}$ without losing generality. The DW is interpreted as the electron-hole pairing condensation [30].

Here, $f_{q}(\boldsymbol{k})$ is given by the Fourier transformation of the spin-independent hopping modulation $\sum_{\boldsymbol{r}_{i}, \boldsymbol{r}_{j}} \delta t_{i, j} e^{i\left(\boldsymbol{r}_{i}-\boldsymbol{r}_{j}\right) \cdot \boldsymbol{k}} e^{i\left(\boldsymbol{r}_{i}+\boldsymbol{r}_{j}\right) \cdot \boldsymbol{q} / 2}$. When $\delta t_{i, j}= \pm \delta t_{j, i}$, the relation $f_{\boldsymbol{q}}(\boldsymbol{k})= \pm f_{\boldsymbol{q}}(-\boldsymbol{k})$ holds. Also, $g_{\boldsymbol{q}}(\boldsymbol{k})$ is given by the spin-dependent modulation $\delta t_{i, j}^{\uparrow}=-\delta t_{i, j}^{\downarrow}$. The even-parity $f_{q}(\boldsymbol{k})$ and the odd-parity $g_{q}(\boldsymbol{k})$ respectively correspond to the BO state and the sLC state. Both states preserve the time-reversal symmetry.

To find possible DWs in an unbiased way, we generalize the DW equation [23] for both spin and charge channels:

$$
\begin{aligned}
& \lambda_{\boldsymbol{q}} f_{\boldsymbol{q}}(k)=-T \sum_{p} I_{\boldsymbol{q}}^{c}(k, p) G\left(p_{-}\right) G\left(p_{+}\right) f_{\boldsymbol{q}}(p), \\
& \eta_{\boldsymbol{q}} g_{\boldsymbol{q}}(k)=-T \sum_{p} I_{q}^{s}(k, p) G\left(p_{-}\right) G\left(p_{+}\right) g_{\boldsymbol{q}}(p),
\end{aligned}
$$

where $\lambda_{q}\left(\eta_{q}\right)$ is the eigenvalue that represents the charge (spin) channel DW instability, $k \equiv\left(\boldsymbol{k}, \epsilon_{n}\right)$, and $p \equiv\left(\boldsymbol{p}, \epsilon_{m}\right)$ $\left(\epsilon_{n}, \epsilon_{m}\right.$ are fermion Matsubara frequencies). These DW equations are interpreted as the "spin/charge channel electron-hole pairing equations."

The charge (spin) channel kernel function is $I_{q}^{c(s)}=$ $I_{q}^{\uparrow, \uparrow}+(-) I_{q}^{\uparrow, \downarrow} ; I_{q}^{\sigma, \rho}$ at $\boldsymbol{q}=0$ is given by the Ward identity $-\delta \Sigma_{\sigma}(k) / \delta G_{\rho}\left(k^{\prime}\right)$, which is composed of one single-magnon exchange term and two double-magnon exchange ones: The former and the latter are called the Maki-Thompson (MT) term and the AL terms; see Fig. 7 in Appendix A. The lowest order Hartree term $-U \delta_{\sigma, \rho}$ in $I_{q}^{\sigma, \rho}$ gives the RPA contribution. while the AL terms are significant for $\alpha_{S} \lesssim 1$ in various strongly correlated systems $[23,35,43,44]$. The significance of the AL processes has been revealed by a functionalrenormalization-group (fRG) study, in which higher-order vertex corrections are produced in an unbiased way $[22,45-$ 47]. Note that the MT term is important for the superconducting gap equation, transport phenomena [40], and CLC order [48].

Figure 2(c) shows the charge-channel eigenvalue $\lambda_{q}$ derived from the DW equation (3) $[23,49,50]$. Hereafter, we put $U$ to satisfy the relation $\alpha_{S}=1-0.444 p^{2}$ with $p \equiv 1-n$, shown as full line in Fig. 2(d). The obtained form factor $f_{\boldsymbol{q}}(\boldsymbol{k})$ at $\boldsymbol{q}=\mathbf{0}, \boldsymbol{Q}_{\mathrm{d}}$, shown in Fig. 2(e), belongs to $B_{1 g}$ symmetry BO, consistent with previous studies [22,23]. As we discuss in Appendix B, the large eigenvalue in Fig. 2(c) [and Fig. 3(a)] is strongly suppressed to $O(1)$ by considering the small quasiparticle weight $z=m / m^{*} \sim O\left(10^{-1}\right)$ due to the self-energy in cuprates $[23,49]$.

\section{DERIVATION OF SLC ORDER BASED ON TRIPLET DW EQUATION}

\section{A. Origin of sLC order}

Next, we discuss the spin-fluctuation-driven sLC order, which is the main issue of this paper. Figure 3(a) exhibits the spin-channel eigenvalue $\eta_{q}$ derived from the DW equation (4). Peaks of $\eta_{\boldsymbol{q}}$ are located at the nesting vectors $\boldsymbol{q}=\boldsymbol{Q}_{\mathrm{d}}$ (diagonal) and $\boldsymbol{q}=\boldsymbol{Q}_{\mathrm{a}}$ (axial). The obtained form factor $g_{\boldsymbol{q}}(\boldsymbol{k})$ at $\boldsymbol{q}=\boldsymbol{Q}_{\mathrm{d}}$ (diagonal sLC) is shown in Fig. 3(b). The odd-parity

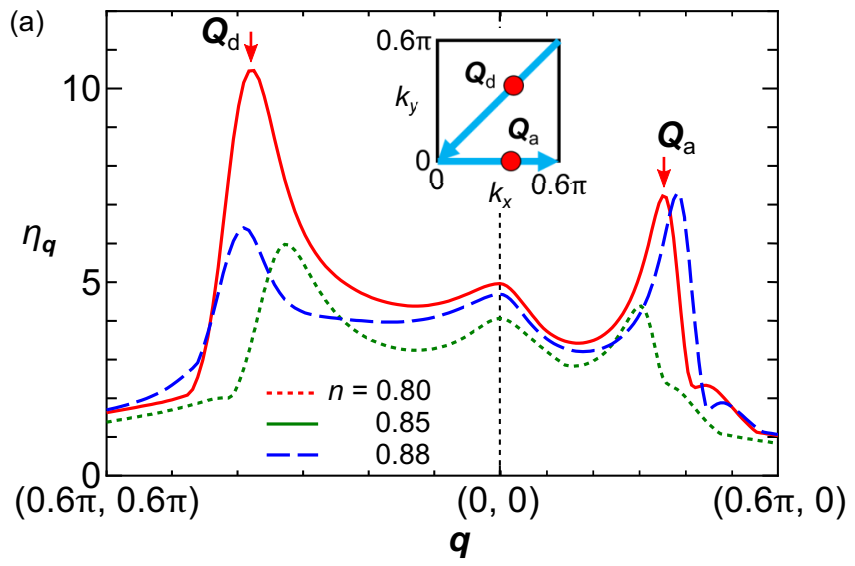

(b)

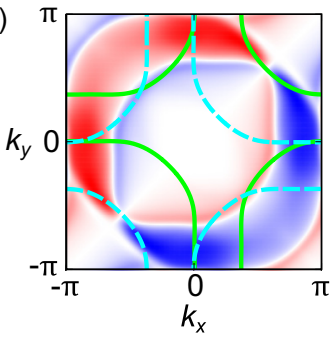

(c)

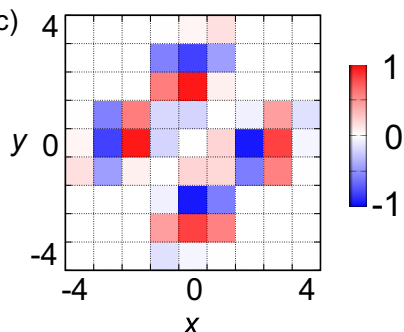

FIG. 3. (a) Obtained eigenvalue $\eta_{q}$ for spin-channel DWs at $n=$ $0.80-0.88$. They have peaks at $\boldsymbol{q}=\boldsymbol{Q}_{\mathrm{d}}$ and $\boldsymbol{Q}_{\mathrm{a}}$. (b) Form factor of the diagonal sLC order $g_{Q_{\mathrm{d}}}(\boldsymbol{k})$. We also show the shifted FSs given by $\mu=\epsilon_{\boldsymbol{k} \pm \boldsymbol{Q}_{\mathrm{d}} / 2}$. (c) $\operatorname{Im} g_{\boldsymbol{Q}_{\mathrm{a}}}(\boldsymbol{r})$, which is even (odd) with respect to $x+y$ $(x-y)$.

solution $g_{q}(\boldsymbol{k})=-g_{q}(-\boldsymbol{k})$ means the emergence of the sLC order. The reason for large $\eta_{Q_{d}}$ is that all hot spots contribute to the diagonal sLC as shown in Fig. 3(b). Figure 3(c) shows the form factor in real space, $\operatorname{Im} g_{\boldsymbol{Q}_{\mathrm{a}}}(\boldsymbol{r})$ with $\boldsymbol{r}=(x, y)$. Here, $\delta t_{i, j}^{\sigma}=\sigma g_{Q_{\mathrm{a}}}\left(\boldsymbol{r}_{i-j}\right) \cos \left(\boldsymbol{r}_{i+j} \cdot \boldsymbol{Q}_{\mathrm{a}} / 2\right)$.

To understand why sLC state is obtained, we simplify Eq. (4) by taking the Matsubara summation analytically by approximating that $I_{q}^{s}$ and $g_{\boldsymbol{q}}(k)$ are static:

$$
\eta_{q} g_{q}(\boldsymbol{k})=\sum_{p} I_{q}^{S}(\boldsymbol{k}, \boldsymbol{p}) F_{q}(\boldsymbol{p}) g_{q}(\boldsymbol{p}),
$$

where

$$
F_{\boldsymbol{q}}(\boldsymbol{p}) \equiv-T \sum_{m} G\left(p_{+}\right) G\left(p_{-}\right)=\frac{n\left(\epsilon_{\boldsymbol{p}_{+}}\right)-n\left(\epsilon_{\boldsymbol{p}_{-}}\right)}{\epsilon_{\boldsymbol{p}_{-}}-\epsilon_{\boldsymbol{p}_{+}}}
$$

is a positive function, and $n(\epsilon)$ is Fermi distribution function; see Appendix A. In general, the peak positions of $\eta_{q}$ in Eq. (5) are located at $\boldsymbol{q}=\mathbf{0}$ and/or nesting vectors with small wavelength $\left(\boldsymbol{q}=\boldsymbol{Q}_{\mathrm{a}}, \boldsymbol{Q}_{\mathrm{d}}\right.$ in the present model). The reason is that $I_{\boldsymbol{q}} \sim T \sum_{p} \chi^{s}\left(\boldsymbol{p}_{+}\right) \chi^{s}\left(\boldsymbol{p}_{-}\right)$by AL terms is large for small $|\boldsymbol{q}|$, and $F_{\boldsymbol{q}}(\boldsymbol{p})$ is large for a wide area of $\boldsymbol{p}$ when $\boldsymbol{q}$ is a nesting vector.

To understand why an odd-parity form factor is obtained, we show the spin-channel "electron-hole pairing interaction" $I_{\boldsymbol{q}=\mathbf{0}}^{s}\left(\boldsymbol{k}, \boldsymbol{k}^{\prime}\right)$ on the FS in Fig. 4(a). The charge-channel one $I_{q=0}^{c}\left(\boldsymbol{k}, \boldsymbol{k}^{\prime}\right)$ is also shown in Fig. 4(b). Here, $\theta$ represents the position of $\boldsymbol{k}$ shown in Figs. 4(c) and 4(d). $I_{q}^{s}\left(\boldsymbol{k}, \boldsymbol{k}^{\prime}\right)$ in Fig. 4(a) gives a large attractive interaction for $\boldsymbol{k} \approx \boldsymbol{k}^{\prime}$ and a large re- 
(a)

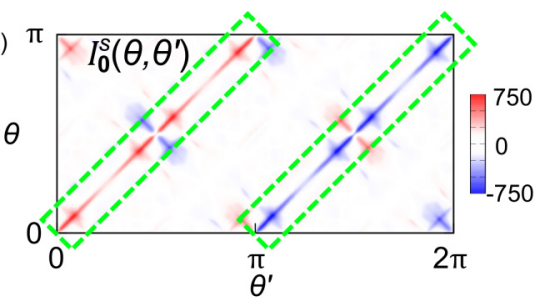

(b)

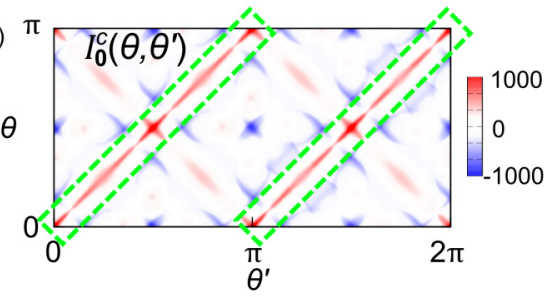

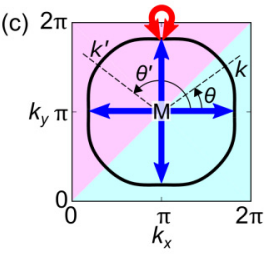

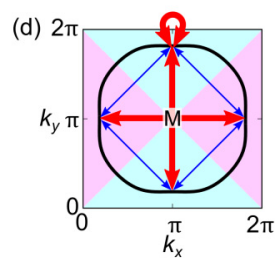

(e)
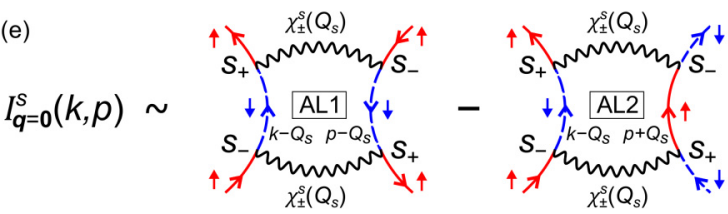

FIG. 4. (a) Spin-channel and (b) charge-channel kernel functions on the FS, $I_{q=0}^{s, c}\left(\theta, \theta^{\prime}\right)$, where $\theta$ represents the position of $\boldsymbol{k}$. We see that $I_{0}^{s}$ and $I_{0}^{c}$ have large negative and positive values for $\theta^{\prime} \approx \theta+\pi$, respectively, due to the p-p channel (= Cooper channel) in AL2. (c) Origin of $p$-wave sLC order and (d) that of $d$-wave BO. Red (blue) color arrows represent the attractive (repulsive) interaction. (e) Spin-flipping AL processes in $I_{q}^{s}(\boldsymbol{k}, \boldsymbol{p})$ that give the sLC order. The wavy lines are transverse spin susceptibilities. (Spin-nonflipping AL processes caused by longitudinal susceptibility are canceled out in $I^{s}=I^{\uparrow, \uparrow}-I^{\uparrow, \downarrow}$.) The AL1 term with an antiparallel (p-h) pair gives the red line on $\theta \approx \theta^{\prime}$ in (a). Also, the AL2 term with a parallel (p-p) pair gives the blue line on $\theta \approx \theta^{\prime}+\pi$ in (a).

pulsive one for $\boldsymbol{k} \approx-\boldsymbol{k}^{\prime}$. In this case, we naturally obtain the $p$-wave form factor $g_{\boldsymbol{q}}(\boldsymbol{k})$ shown in Fig. 3(b), as we explain in Fig. 4(c). Here, red (blue) arrows represent the attractive (repulsive) interaction.

The strong $\boldsymbol{k}, \boldsymbol{k}^{\prime}$ dependence of $I_{\boldsymbol{q}=\mathbf{0}}^{s}\left(\boldsymbol{k}, \boldsymbol{k}^{\prime}\right)$ originates from the AL1 and AL2 terms in Fig. 4(e), or Fig. 7(a) in Appendix A. Owing to the spin-conservation law, AL terms in $I^{s}=I^{\uparrow, \uparrow}-I^{\uparrow, \downarrow}$ originate from the spin-flipping processes due to transverse spin fluctuations in Fig. 4(e), in proportion to $\chi_{ \pm}^{s}\left(\boldsymbol{Q}_{s}\right) \chi_{ \pm}^{s}\left(\boldsymbol{Q}_{s}\right)$. [In $I^{s}$, the spin non-flipping AL processes in proportion to $\chi_{z}^{s}\left(\boldsymbol{Q}_{s}\right) \chi_{z}^{s}\left(\boldsymbol{Q}_{s}\right)$ are exactly canceled out.] Therefore, $I^{s}=[\mathrm{AL} 1]-[\mathrm{AL} 2]$. The AL1 term with the p$\mathrm{h}$ (antiparallel) pair Green functions causes large attractive interaction for $\boldsymbol{k} \approx \boldsymbol{k}^{\prime}$, and the AL2 term with the p-p (parallel) ones does so for $\boldsymbol{k} \approx-\boldsymbol{k}^{\prime}$, as explained in Ref. [49] in detail. Thus, $\theta, \theta^{\prime}$ dependence in Fig. 4(a) and the resultant odd-parity solution is understood naturally.

In contrast, the charge channel kernel $I_{\boldsymbol{q}}^{c}\left(\boldsymbol{k}, \boldsymbol{k}^{\prime}\right)$ gives an attractive interaction for both $\boldsymbol{k} \approx \pm \boldsymbol{k}^{\prime}$ as shown in Fig. 4(b), because $I^{c}=3([\mathrm{AL} 1]+[\mathrm{AL} 2]) / 2$. Then, we obtain the $d$ wave form factor $f_{\boldsymbol{q}}(\boldsymbol{k})$ in Fig. 2(e), as we explain in Fig. 4(d) $[23,49]$.

In the present transverse spin fluctuation mechanism, the $g$ vector will be parallel to $z$ direction when $\chi_{x(y)}^{s}\left(\boldsymbol{Q}_{s}\right)>\chi_{z}^{s}\left(\boldsymbol{Q}_{s}\right)$ ( $X Y$ anisotropy) due to the spin-orbit interaction (SOI). When
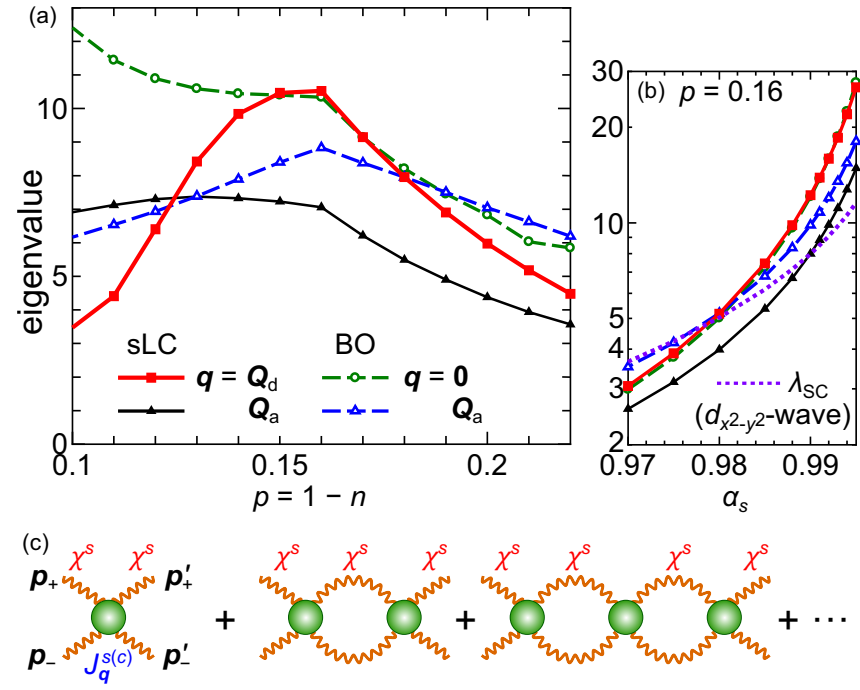

FIG. 5. (a) Obtained eigenvalues of sLC and BO as functions of $p=1-n$. (b) $\alpha_{S}$ dependences of the eigenvalues at $p=0.16$. $\lambda_{\mathrm{SC}}$ is the eigenvalue of the superconducting gap equation. (c) Diagrammatic expression for the odd/even parity magnon-pair condensation, which is the physical origin of the sLC/BO.

the $X Y$ anisotropy of $\chi_{\mu}^{s}\left(\boldsymbol{Q}_{s}\right)$ is very large, $I^{c}$ due to AL terms is multiplied by $2 / 3$ whereas $I^{s}$ is unchanged, so it is suitable condition for the sLC order.

\section{B. Filling dependences of $\mathrm{SLC} / \mathrm{BO}$ instabilities}

The sLC and BO eigenvalues are summarized in Fig. 5(a). The relation $\eta_{Q_{\mathrm{d}}}>\lambda_{Q_{\mathrm{a}}}$ around the optimal doping $(p \sim 0.15)$ means that the sLC transition temperature $T_{\mathrm{SLC}}$ is higher than $T_{\mathrm{CDW}}$, as in Fig. 1(a). The robustness of Fig. 5(a) is verified in Appendix B. We also verify in Fig. 5(b) that both $\eta_{\boldsymbol{Q}_{\mathrm{d}}}$ and $\lambda_{\boldsymbol{Q}_{\mathrm{a}}}$ are larger than the $d_{x^{2}-y^{2}}$-wave superconducting eigenvalue $\lambda_{\mathrm{SC}}$ for $\alpha_{S} \gtrsim 0.98$. Here, $\lambda_{\mathrm{SC}}$ is derived from the gap equation

$$
\lambda_{\mathrm{SC}} \Delta(k)=T \sum_{p} V^{\mathrm{SC}}(k, p)|G(p)|^{2} \Delta(p),
$$

where $V^{\mathrm{SC}}(k, p)=-\frac{3}{2} U^{2} \chi^{s}(k-p)+\frac{1}{2} U^{2} \chi^{c}(k-p)-U$ is the MT-type kernel [51]. Note that $\eta_{\boldsymbol{q}}, \lambda_{\boldsymbol{q}}<\lambda_{\mathrm{SC}}$ if AL terms are dropped [52]. The large eigenvalues in Fig. 5 are suppressed to $O(1)$ by small $z$; see Refs. [23,40,49] and Appendix B. We stress that sLC is not suppressed by the ferro-BO that induces neither Fermi arc nor pseudogap, as explained in Appendix B.

Here, we analyzed the sLC/BO in terms of the electronhole pairing. Another physical interpretation of the sLC/BO is the "condensation of odd/even parity magnon pairs," which is the origin of the nematic order in quantum spin systems [53-55]. In fact, the two-magnon propagator shown in Fig. 5(c) diverges when the eigenvalue of DW equation reaches unity, as we explain in Appendix C. That is, triplet (singlet) magnon-pair condensation occurs at $T=$ $T_{\mathrm{sLC}}\left(T_{\mathrm{CDW}}\right)$. Thus, the sLC/BO discussed here and the spin nematic order in quantum spin systems are essentially the same phenomenon. 


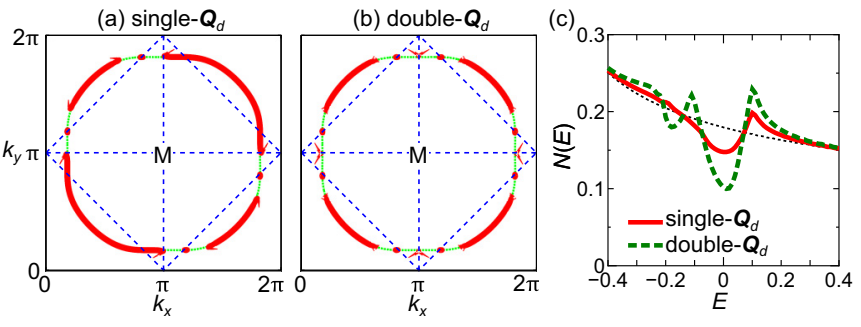

FIG. 6. (a) Fermi arc structure due to the single- $\boldsymbol{q}$ order, (b) that due to the double- $\boldsymbol{q}$ order, and (c) pseudogap in the DOS due to the diagonal sLC order $\left(\boldsymbol{q}=\boldsymbol{Q}_{\mathrm{d}}\right)$. In calculating (a)-(c), we introduced BCS-type cutoff energy $\omega_{c}=0.5$ for the band hybridization by $g_{q}(\boldsymbol{k})$.

\section{Fermi arc and pseudogap under sLC order}

Now, we discuss the band-folding and hybridization gap due to the diagonal sLC order. Figures 6(a) and 6(b) show the Fermi arc structures in the cases of (a) single- $q$ and (b) double- $\boldsymbol{q}$ orders. We set $g^{\max } \equiv \max _{\boldsymbol{k}}\left\{g_{\boldsymbol{Q}_{\mathrm{d}}}(\boldsymbol{k})\right\}=0.1$. Here, the folded band structure under the sLC order with finite $\boldsymbol{q}_{\mathrm{sLC}}$ is "unfolded" into the original Brillouin zone by following Ref. [56] to make a comparison with angle-resolved photoemission spectroscopy (ARPES) results. The Fermi arc due to the single- $\boldsymbol{q}$ order in Fig. 6(a) belongs to $B_{2 g}$ symmetry. In contrast, the Fermi arc due to the double- $q$ order in Fig. 6(b) preserves the $C_{4}$ symmetry. The resultant pseudogap in the DOS is shown in Fig. 6(c). The unfolded band structure in the single- $\boldsymbol{Q}_{\mathrm{d}} \mathrm{sLC}$ order is displayed in Fig. 12 in Appendix B.

Recent magnetic torque measurements revealed that the $B_{1 g}$ symmetry breaking at $T=T^{*}$ occurs in YBCO [10], while the $B_{2 g}$ one appears in Hg-based cuprates [11]. To understand different symmetry breakings, we examine the $t_{3}$ dependence of the DW equation solution in Appendix B 2: As shown in Figs. 9(b)-9(e), the sLC wave vector $\boldsymbol{q}_{\mathrm{sLC}}$ changes from $\boldsymbol{Q}_{\mathrm{d}}$ to $\boldsymbol{Q}_{\mathrm{a}}$ for larger $t_{3} / t_{1}$. When $\boldsymbol{q}_{\mathrm{sLC}}=\boldsymbol{Q}_{\mathrm{d}}$, the symmetry of the Fermi arc is $B_{2 g}$ in Fig. 6(a). On the other hand, the Fermi arc has $B_{2 g}$ symmetry in the axial sLC order in Fig. 1(d), as we show in Fig. 10(b).

Since the van Vleck susceptibility becomes anisotropic when $C_{4}$ symmetry of the FS is broken [57], the reported compound-dependent symmetry breaking $[10,11]$ would be explained by the sLC order scenario. This is an important future issue.

\section{Spin-current pattern under sLC order}

Next, we investigate the spin current in real space, which is driven by a fictitious Peierls phase due to the "spindependent self-energy" $\delta t_{i, j}^{\sigma}=\sigma g_{i, j}$. As shown in Fig. 3(c), $\delta t_{i, j}$ is purely imaginary and odd with respect to $i \leftrightarrow j$. The conservation law $\dot{n}_{i}^{\sigma}=\sum_{j} j_{i, j}^{\sigma}$ directly leads to the definition the spin current operator from site $j$ to site $i$ as $j_{i, j}^{\sigma}=-i \sum_{\sigma} \sigma\left(h_{i, j}^{\sigma} c_{i \sigma}^{\dagger} c_{j \sigma}-(i \leftrightarrow j)\right)$, where $h_{i, j}^{\sigma}=t_{i, j}+$ $\delta t_{i, j}^{\sigma}$. Then, the spontaneous spin current from $j$ to $i$ is $J_{i, j}^{s}=$ $\left\langle j_{i, j}^{s}\right\rangle_{\hat{h}^{\sigma}}$.

Here, we calculate the spin current for the commensurate sLC order at $\boldsymbol{q}_{\mathrm{sLC}}=(\pi / 2, \pi / 2)$, which is achieved by putting $n=1.0$. Then, the unit cells are composed of four sites A-D.
In Fig. 14 in Appendix D, we show the obtained spin current $J_{i, j}^{s}$ from the center site $(j=\mathrm{A}-\mathrm{B})$ to a different site in Fig. 1(c). The derived spin current pattern between the nearest and second-nearest sites is depicted in Fig. 1(c).

The Fermi arc structure and the DOS in Fig. 6 are independent of the phase shift $g_{q} \rightarrow e^{i \psi} g_{q}$. In contrast, the real space current pattern depends on the phase shift. We discuss other possible sLC patterns in Appendix D. The charge modulation due to the sLC is just $\left|\Delta n_{i}\right| \sim 5 \times 10^{-4}$ for $g^{\max }=0.1$ since $\left|\Delta n_{i}\right| \propto\left(g^{\max }\right)^{2}$. Thus, experimental detection of translational symmetry breaking by sLC order may be difficult. However, the cLC is induced by applying a uniform magnetic field parallel to $\boldsymbol{g}_{i, j}$. In the present sLC state, under $10 \mathrm{~T}$ magnetic field, the induced cLC gives $\Delta H \sim \pm 0.1$ Oe when $m^{*} / m \sim$ 10 , which may be measurable by nuclear magnetic resonance (NMR) or muon spin resonance ( $\mu \mathrm{SR}$ ) study.

\section{E. sLC fluctuation mediated superconductivity}

Finally, we discuss the result that the sLC fluctuations can contribute to the $d_{x^{2}-y^{2}}$-wave pairing mechanism. The sLC fluctuations connect the close hot spots at $\boldsymbol{P}$ and $\boldsymbol{P}^{\prime}=\boldsymbol{P}+\boldsymbol{q}$ in Fig. 2(a). At both points, the $d_{x^{2}-y^{2}}$-wave gap function $\Delta_{k}$ has the same sign. The pairing interaction mediated by the spin-channel sLC fluctuations between singlet pairs $(\boldsymbol{P},-\boldsymbol{P})$ and $\left(\boldsymbol{P}^{\prime},-\boldsymbol{P}^{\prime}\right)$ is

$$
V^{\mathrm{sLC}}\left(\boldsymbol{P}, \boldsymbol{P}^{\prime}\right) \propto g_{\boldsymbol{q}}(\boldsymbol{k}) g_{\boldsymbol{q}}(-\boldsymbol{k}) \cdot\left[-\chi_{\mathrm{sLC}}(\boldsymbol{q})\right],
$$

where $\boldsymbol{k}=\left(\boldsymbol{P}+\boldsymbol{P}^{\prime}\right) / 2$ and $\boldsymbol{q}=\boldsymbol{P}-\boldsymbol{P}^{\prime} \sim \boldsymbol{Q}_{\mathrm{a}} \cdot \chi_{\mathrm{SLC}}(\boldsymbol{q})(>0)$ is the sLC susceptibility and $g_{q}(\boldsymbol{k})=-g_{\boldsymbol{q}}(-\boldsymbol{k})$ is its form factor. Thus, Eq. (7) give a positive (i.e., attractive) pairing interaction between close hot spots. The derivation of Eq. (7) is given in Appendix A and in Supplemental Material Sec. F of Ref. [50]. This mechanism may be important for slightly overdoped cuprates with $T_{\text {sLC }} \lesssim T_{\mathrm{c}}$.

\section{SUMMARY}

In summary, we proposed a long-sought formation mechanism for the nanoscale spin-current order, which violates the parity and translational symmetry while time-reversal symmetry is preserved. It was revealed that the formation of triplet odd-parity electron-hole pairs is mediated by spin fluctuations, and therefore the spontaneous sLC is established at $T=T_{\mathrm{sLC}}$. In the present spin-fluctuation mechanism, the condensation of odd-parity magnon pairs occurs simultaneously. The band folding by the sLC orders results in the formations of the Fermi arc structure and pseudogap at $T \sim T^{*}$. In the sLC state, a staggered moment is expected to appear under a uniform magnetic field. The sLC order will be a key ingredient in understanding pseudogap phase and electronic nematicity not only in cuprates, but also in iridates $[32,39]$ and heavy-fermion compounds. It is an important future issue to incorporate the self-energy effect into the present theory.

\section{ACKNOWLEDGMENTS}

The authors are grateful to Y. Matsuda, K. Yamada, T. Moriya, and A. Kobayashi for fruitful comments and discussions. This work was supported by the project "Quantum 
Liquid Crystals" No. JP19H05825 KAKENHI on Innovative Areas from JSPS of Japan, and by JSPS KAKENHI (JP18H01175, JP20K22328, JP20K03858, JP17K05543).

\section{APPENDIX A: DERIVATION OF SINGLET AND TRIPLET DW EQUATIONS}

Here, we discuss the linearized density-wave (DW) equation driven by spin fluctuations. For this purpose, we introduce the irreducible four-point vertex function $I_{q}^{\sigma, \rho}\left(k, k^{\prime}\right)$. It is given by the Ward identity at $\boldsymbol{q}=0$, that is, $I_{q}^{\sigma, \rho}\left(k, k^{\prime}\right) \equiv$ $-\delta \Sigma_{\sigma}(k) / \delta G_{\rho}\left(k^{\prime}\right)$. Here, we use the one-loop self-energy given as

$$
\begin{aligned}
\Sigma_{\sigma}(k)= & T \sum_{q} U^{2} \chi_{\mathrm{L}}^{\sigma}(q) G_{\sigma}(k+q) \\
& +T \sum_{p} U^{2}\left(\chi_{\mathrm{T}}^{\sigma}(q)-\chi_{\mathrm{T}}^{(0) \sigma}(q)\right) G_{-\sigma}(k+q),
\end{aligned}
$$

where $\chi_{\mathrm{L}}^{\sigma}(q)$ and $\chi_{\mathrm{T}}^{\sigma}(q)$ are longitudinal and transverse susceptibilities. They are given as

$$
\begin{gathered}
\chi_{\mathrm{L}}^{\sigma}(q)=\chi_{\mathrm{L}}^{(0) \sigma}(q)\left(1-U^{2} \chi_{\mathrm{L}}^{(0) \sigma}(q) \chi_{\mathrm{L}}^{(0)-\sigma}(q)\right)^{-1}, \\
\chi_{\mathrm{T}}^{\sigma}(q)=\chi_{\mathrm{T}}^{(0) \sigma}(q)\left(1-U \chi_{\mathrm{T}}^{(0) \sigma}(q)\right)^{-1},
\end{gathered}
$$

where $\chi_{\mathrm{L}}^{(0) \sigma}(q)=-T \sum_{p} G_{\sigma}(p) G_{\sigma}(p+q)$ and $\chi_{\mathrm{T}}^{(0) \sigma}(q)=$ $-T \sum_{p} G_{\sigma}(p) G_{-\sigma}(p+q)$ are longitudinal and transverse irreducible susceptibilities. Then, the irreducible vertex function $I_{q}^{\sigma, \rho}\left(k, k^{\prime}\right)$ given by the Ward identity is composed of one MT term and two AL terms in Fig. 7(a). Note that $I_{q}^{\sigma, \rho}$ contains the lowest order Hartree term $-U \delta_{\sigma, \rho}$.

First, we derive the charge-channel (singlet) DW equation in the absence of the magnetic field, where the form factor is independent of spin: $f_{\boldsymbol{q}}^{\uparrow}(k)=f_{\boldsymbol{q}}^{\downarrow}(k)=f_{\boldsymbol{q}}(k)$. The singlet DW equation was introduced in the study of Fe-based superconductors [49] and cuprate superconductors [23]. It is given as

$$
\lambda_{\boldsymbol{q}} f_{\boldsymbol{q}}(k)=-T \sum_{k^{\prime}} I_{\boldsymbol{q}}^{c}\left(k, k^{\prime}\right) G\left(k_{-}^{\prime}\right) G\left(k_{+}^{\prime}\right) f_{\boldsymbol{q}}\left(k^{\prime}\right),
$$

which is shown in Fig. 7(b), and $\boldsymbol{k}_{ \pm} \equiv \boldsymbol{k} \pm \boldsymbol{q} / 2$. Here, $I_{\boldsymbol{q}}^{c}\left(k, k^{\prime}\right)=I_{\boldsymbol{q}}^{\sigma, \sigma}\left(k, k^{\prime}\right)+I_{\boldsymbol{q}}^{\sigma,-\sigma}\left(k, k^{\prime}\right)$. It is given as

$$
\begin{aligned}
I_{q}^{c}\left(k, k^{\prime}\right)= & -\frac{3}{2} V^{s}\left(k-k^{\prime}\right)-\frac{1}{2} V^{c}\left(k-k^{\prime}\right) \\
& +T \sum_{p}\left[\frac{3}{2} V^{s}\left(p_{+}\right) V^{s}\left(p_{-}\right)+\frac{1}{2} V^{c}\left(p_{+}\right) V^{c}\left(p_{-}\right)\right] \\
& \times G(k-p) G\left(k^{\prime}-p\right) \\
& +T \sum_{p}\left[\frac{3}{2} V^{s}\left(p_{+}\right) V^{s}\left(p_{-}\right)+\frac{1}{2} V^{c}\left(p_{+}\right) V^{c}\left(p_{-}\right)\right] \\
& \times G(k-p) G\left(k^{\prime}+p\right),
\end{aligned}
$$

where $p=\left(\boldsymbol{p}, \omega_{l}\right), \quad \hat{V}^{s}(q)=U+U^{2} \hat{\chi}^{s}(q)$, and $\hat{V}^{c}(q)=$ $-U+U^{2} \hat{\chi}^{c}(q)$. The first, the second, the third terms in Eq. (A5) corresponds to the MT, AL1, and AL2 terms in Fig. 7(a).
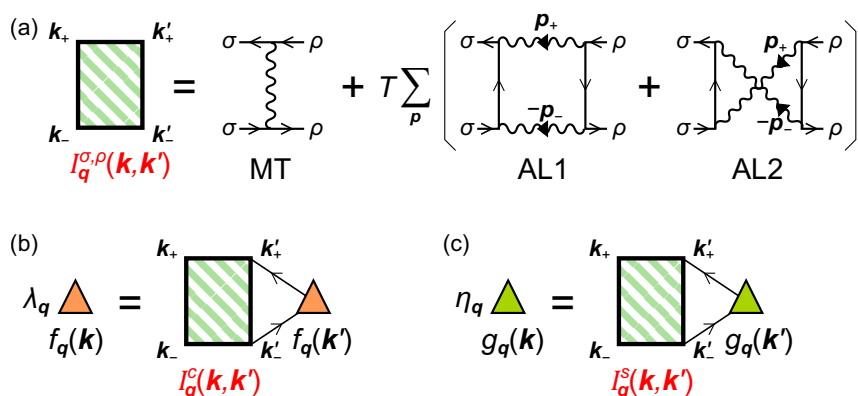

(d)
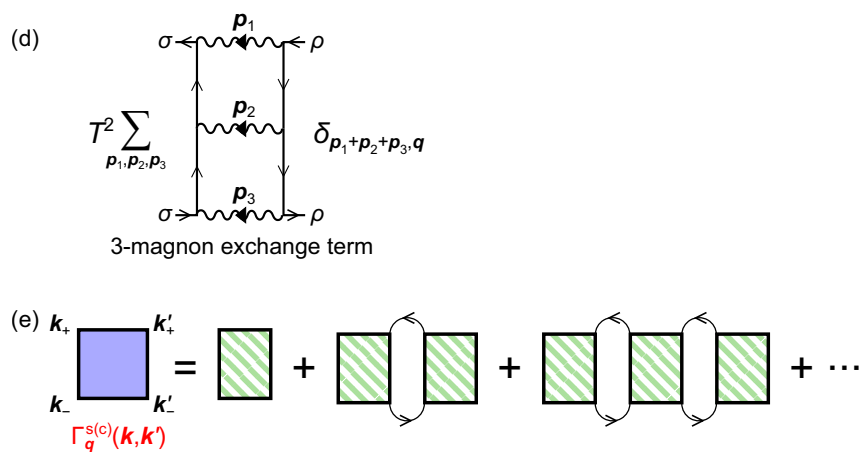

FIG. 7. (a) Irreducible four-point vertex $I_{q}^{\sigma, \rho}\left(k, k^{\prime}\right)$ composed of one MT term and two AL terms. (b) Linearized singlet DW equation with the kernel $I^{c} \equiv I^{\sigma, \sigma}+I^{\sigma,-\sigma}$. (c) Linearized triplet DW equation with the kernel $I^{s} \equiv I^{\sigma, \sigma}-I^{\sigma,-\sigma}$. (d) A three-magnon exchange term, which is less important. (e) Full four-point vertex function $\Gamma_{q}^{s(c)}\left(k, k^{\prime}\right)$ given by solving the DW equation. The sLC order (BO) emerges when $\Gamma_{\boldsymbol{q}}^{s(c)}\left(k, k^{\prime}\right)$ diverges.

In cuprates, Eq. (A4) gives an even-parity solution with wave vector $\boldsymbol{q}=\mathbf{0}$ and $\boldsymbol{q} \approx(\pi / 2,0)$. This singlet and evenparity electron-hole condensation is interpreted as the BO.

Next, we derive the spin-channel (triplet) DW equation in the absence of the magnetic field, the spin-dependent form factor is $g_{\boldsymbol{q}}(k) \equiv g_{\boldsymbol{q}}^{\uparrow}(k)=-g_{\boldsymbol{q}}^{\downarrow}(k)$. It is given as

$$
\eta_{\boldsymbol{q}} g_{\boldsymbol{q}}(k)=-T \sum_{k^{\prime}} I_{\boldsymbol{q}}^{s}\left(k, k^{\prime}\right) G\left(k_{-}^{\prime}\right) G\left(k_{+}^{\prime}\right) g_{\boldsymbol{q}}\left(k^{\prime}\right),
$$

which is shown in Fig. 7 (c). Here, $I_{q}^{s}\left(k, k^{\prime}\right)=I_{q}^{\sigma, \sigma}\left(k, k^{\prime}\right)-$ $I_{q}^{\sigma,-\sigma}\left(k, k^{\prime}\right)$. It is given as

$$
\begin{aligned}
I_{q}^{s}\left(k, k^{\prime}\right)= & \frac{1}{2} V^{s}\left(k-k^{\prime}\right)-\frac{1}{2} V^{c}\left(k-k^{\prime}\right) \\
& +T \sum_{p}\left[V^{s}\left(p_{+}\right) V^{s}\left(p_{-}\right)+\frac{1}{2} V^{s}\left(p_{+}\right) V^{c}\left(p_{-}\right)\right. \\
& \left.+\frac{1}{2} V^{c}\left(p_{+}\right) V^{s}\left(p_{-}\right)\right] G(k-p) G\left(k^{\prime}-p\right) \\
& +T \sum_{p}\left[-V^{s}\left(p_{+}\right) V^{s}\left(p_{-}\right)+\frac{1}{2} V^{s}\left(p_{+}\right) V^{c}\left(p_{-}\right)\right. \\
& \left.+\frac{1}{2} V^{c}\left(p_{+}\right) V^{s}\left(p_{-}\right)\right] G(k-p) G\left(k^{\prime}+p\right), \quad \text { (A7) }
\end{aligned}
$$

where the first, second, and third terms in Eq. (A5) correspond to the MT, AL1, and AL2 terms in Fig. 7(a). The AL terms with $V^{s}\left(p_{+}\right) V^{s}\left(p_{-}\right)$are shown in Fig. 4 (e). In cuprates, 
Eq. (A6) gives the odd-parity solution at wave vectors $\boldsymbol{q}=$ $(\pi / 2, \pi / 2)$ and $(\pi / 2,0)$. This triplet and odd-parity electronhole pairing is interpreted as the spin-loop current (sLC).

In both Eqs. (A5) and (A7), the AL terms are proportional to $\phi_{q}^{(2)} \equiv T \sum_{\boldsymbol{p}_{1}, \boldsymbol{p}_{2}} V^{s}\left(\boldsymbol{p}_{1}\right) V^{s}\left(\boldsymbol{p}_{2}\right) \cdot \delta_{\boldsymbol{p}_{1}+\boldsymbol{p}_{2}, \boldsymbol{q}}$. The AL terms are significant when the spin fluctuations are large, since both $V^{s}\left(\boldsymbol{p}_{1}\right)$ and $V^{s}\left(\boldsymbol{q}-\boldsymbol{p}_{1}\right)$ take large value simultaneously when $\boldsymbol{p}_{1} \approx \boldsymbol{Q}_{s}$ in the case of $\boldsymbol{q} \approx \mathbf{0}$. If we put $V^{s}(\boldsymbol{p}) \propto \xi^{2} /[1+$ $\left.\xi^{2}\left(\boldsymbol{p}-\boldsymbol{Q}_{s}\right)^{2}\right]$ at zero Matsubara frequency, where $\xi(\gg$ $1)$ is the magnetic correlation length, $\phi_{q=0}^{(2)} \propto T \xi^{2}$ in twodimensional systems. Therefore, double-magnon exchange (AL) terms induce not only $\mathrm{BO}$, but also the sLC order when $\xi \gg 1$. A three-magnon exchange term shown in Fig. 7(d) is proportional to $\phi_{q}^{(3)} \equiv T^{2} \sum_{\boldsymbol{p}_{1}, \boldsymbol{p}_{2}, \boldsymbol{p}_{3}} V^{s}\left(\boldsymbol{p}_{1}\right) V^{s}\left(\boldsymbol{p}_{2}\right) V^{s}\left(\boldsymbol{p}_{3}\right)$. $\delta_{p_{1}+p_{2}+p_{3}, q}$. Then, $\phi_{q=0}^{(3)} \propto T^{2} \xi^{2}$ in two-dimensional systems for $\boldsymbol{q} \sim \boldsymbol{Q}_{s}$, which is smaller than $\phi_{\boldsymbol{q}=\mathbf{0}}^{(2)}$ at low temperatures $T \ll E_{\mathrm{F}}$. Thus, the AL process would be the most significant, which is also indicated by functional-renormalization-group studies [22].

The electron-hole pairing order is generally expressed in real space as follows [30]:

$$
\begin{aligned}
D_{i, j}^{\sigma, \rho} & \equiv\left\langle c_{i \sigma}^{\dagger} c_{j \rho}\right\rangle-\left\langle c_{i \sigma}^{\dagger} c_{j \rho}\right\rangle_{0} \\
& =d_{i, j}^{c} \delta_{\sigma, \rho}+\boldsymbol{d}_{i, j}^{s} \cdot \boldsymbol{\sigma}_{\sigma, \rho},
\end{aligned}
$$

where $D_{i, j}^{\sigma, \rho}=\left\{D_{j, i}^{\rho, \sigma}\right\}^{*}$, and $d_{i, j}^{c}\left[\boldsymbol{d}_{i, j}^{s}\right]$ is the spin singlet (triplet) pairing. It induces the symmetry breaking in the selfenergy:

$$
\Delta \Sigma_{i, j}^{\sigma \rho}=f_{i, j} \delta_{\sigma, \rho}+\boldsymbol{g}_{i, j} \cdot \boldsymbol{\sigma}_{\sigma, \rho}
$$

which we call the form factors in this paper. The $\mathrm{BO}$ is given by real even-parity function $f_{i, j}=f_{j, i}$, and the sLC is given by pure imaginary odd-parity vector $\boldsymbol{g}_{i, j}=-\boldsymbol{g}_{j, i}$. Both orders preserve the time-reversal symmetry. Note that $f_{\boldsymbol{q}}(k)$ and $g_{\boldsymbol{q}}(k)$ in Eqs. (A4) and (A6) correspond to $f_{i, j}$ and $g_{i, j}^{z}$, respectively.

Finally, we discuss the effective interaction driven by the $\mathrm{BO} / \mathrm{sLC}$ fluctuations. By solving the DW equation (A4), we obtain the full four-point vertex function $\Gamma_{q}^{c}\left(k, k^{\prime}\right)$ that is composed of $I_{\boldsymbol{q}}^{c}$ and $G\left(k_{+}\right) G\left(k_{-}\right)$shown in Fig. 7(e), which increases in proportion to $\left(1-\eta_{q}\right)^{-1}$. Thus, we obtain the relation $\Gamma_{\boldsymbol{q}}^{c}\left(k, k^{\prime}\right) \approx f_{\boldsymbol{q}}(k)\left\{f_{\boldsymbol{q}}\left(k^{\prime}\right)\right\}^{*} \bar{I}_{\boldsymbol{q}}^{c}\left(1-\lambda_{\boldsymbol{q}}\right)^{-1}$, which is well satisfied when $\lambda_{q}$ is close to unity. Here, $\bar{I}_{q}^{c(s)} \equiv$ $T^{2} \sum_{k, k^{\prime}}\left\{f_{\boldsymbol{q}}(k)\right\}^{*} I_{\boldsymbol{q}}^{c(s)}\left(k, k^{\prime}\right) f_{\boldsymbol{q}}\left(k^{\prime}\right) / T \sum_{k}\left|f_{\boldsymbol{q}}(k)\right|^{2}$. In the same way, we obtain the relation $\Gamma_{\boldsymbol{q}}^{s}\left(k, k^{\prime}\right) \approx g_{\boldsymbol{q}}(k)\left\{g_{\boldsymbol{q}}\left(k^{\prime}\right)\right\}^{*} \bar{I}_{\boldsymbol{q}}^{s}(1-$ $\left.\eta_{q}\right)^{-1}$. Thus, it is apparent that the sLC order $g$ (BO $f$ ) emerges when $\Gamma_{q}^{s(c)}\left(k, k^{\prime}\right)$ diverges.

The pairing interaction due to the sLC fluctuations is given by the full four-point vertex. It is approximately expressed as $V^{\mathrm{SC}}\left(\boldsymbol{k}_{+}, \boldsymbol{k}_{-}\right)=-\Gamma_{q}^{s}(\boldsymbol{k},-\boldsymbol{k}) \propto-g_{\boldsymbol{q}}(\boldsymbol{k})\left\{g_{\boldsymbol{q}}(-\boldsymbol{k})\right\}^{*}\left(1-\eta_{\boldsymbol{q}}\right)^{-1}$. Since $g$ is an odd function, the sLC fluctuations cause attractive interaction: $V^{\mathrm{SC}}\left(\boldsymbol{k}_{+}, \boldsymbol{k}_{-}\right) \propto-\left|g_{\boldsymbol{q}}(\boldsymbol{k})\right|^{2}\left(1-\eta_{\boldsymbol{q}}\right)^{-1}$.

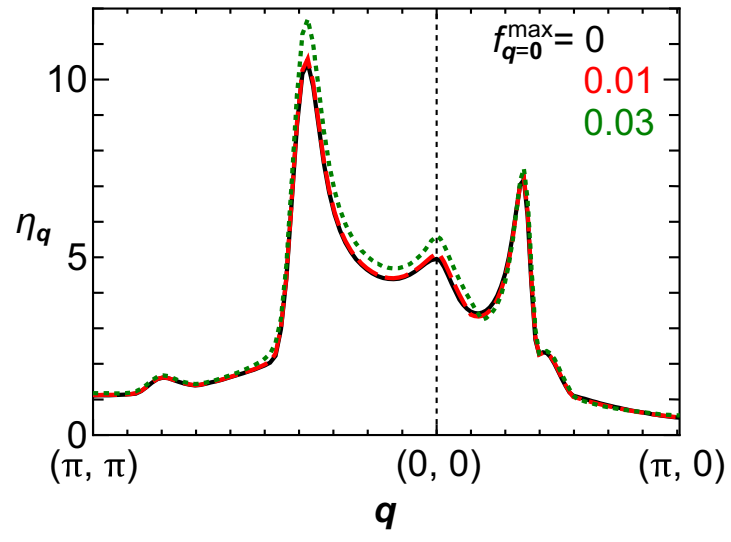

FIG. 8. Obtained $\eta_{q}$ for $n=0.85\left(\alpha_{S}=0.99\right)$ under the ferroBO with $f_{q=\mathbf{0}}^{\max }=0,0.01,0.03$. Thus, the ferro-BO does not prohibit the emergence of the sLC order.

\section{APPENDIX B: ADDITIONAL NUMERICAL RESULTS OF DW EQUATIONS}

\section{Enhancement of the sLC instability under the finite ferro-BO}

In Figs. 5(a) and 5(b) in the main text, the sLC eigenvalue $\eta_{q=Q_{\mathrm{d}}}$ is comparable to the BO eigenvalue $\lambda_{q=0}$ for a wide doping range. This result means that the sLC order at $\boldsymbol{q}_{\mathrm{sLC}}=$ $Q_{\mathrm{d}}$ and the ferro-BO occur at almost the same temperature $\sim T^{*}$. Here, we discuss the possibility of coexistence of sLC order and ferro-BO.

Since the ferro-BO does not induce the band folding and pseudogap, the sLC order will emerge even if the ferro-BO transiting temperature is higher. To verify this expectation, we calculated the triplet DW equation (A6) under the ferro-BO with $f_{q=0}^{\max }=0,0.01,0.03$. Figure 8 shows the eigenvalue of sLC as function of $\boldsymbol{q}$ for $n=0.85$ and $U=3.27\left(\alpha_{S}=0.99\right)$ under the ferro-BO form factor obtained by the spin-singlet DW equation (A4). It is verified that the ferro-BO does not prohibit the emergence of the sLC order. The eigenvalue $\eta_{q}$ slightly increases with $f_{q=0}^{\max }$, since the spin Stoner factor $\alpha_{S}$ is enlarged by the ferro-BO $[22,23]$.

\section{Change in the phase diagram by $t_{3}$}

In the main text, we show that the sLC eigenvalue at $\boldsymbol{q}=\boldsymbol{Q}_{\mathrm{d}}$ develops as large as the ferro-BO eigenvalue near the optimal-doping case ( $p \sim 0.15$ ), based on the Hubbard model with the hopping integrals $\left(t_{1}, t_{2}, t_{3}\right)=(-1.0,1 / 6,-1 / 5)$. The obtained Fermi surface (FS) has the flat part near the Brillouin zone boundary, which captures the characteristic of YBCO compounds.

Here, we examine a key model parameter for the phase diagram, and reveal that the sLC instability is sensitively controlled by $t_{3}$. Figure 9(a) shows the FSs for $t_{3}=-0.20$ to 0.25 in the case of $t_{1}=-1.0$ and $t_{2}=1 / 6$. The shape of the flat part of the FS near the BZ, which is significant for the density wave (DW) instabilities at $\boldsymbol{q}=\boldsymbol{Q}_{\mathrm{a}}, \boldsymbol{Q}_{\mathrm{d}}$, is sensitively modified by $t_{3}$.

Figures 9(b)-9(e) show the obtained spin-channel eigenvalue $\eta_{q}$ and the charge-channel one $\lambda_{q}$ in the cases of $t_{3}=$ $-0.23[(\mathrm{~b})$ and (c) $]$ and $t_{3}=-0.25[(\mathrm{~d})$ and (e)]. (We set the 

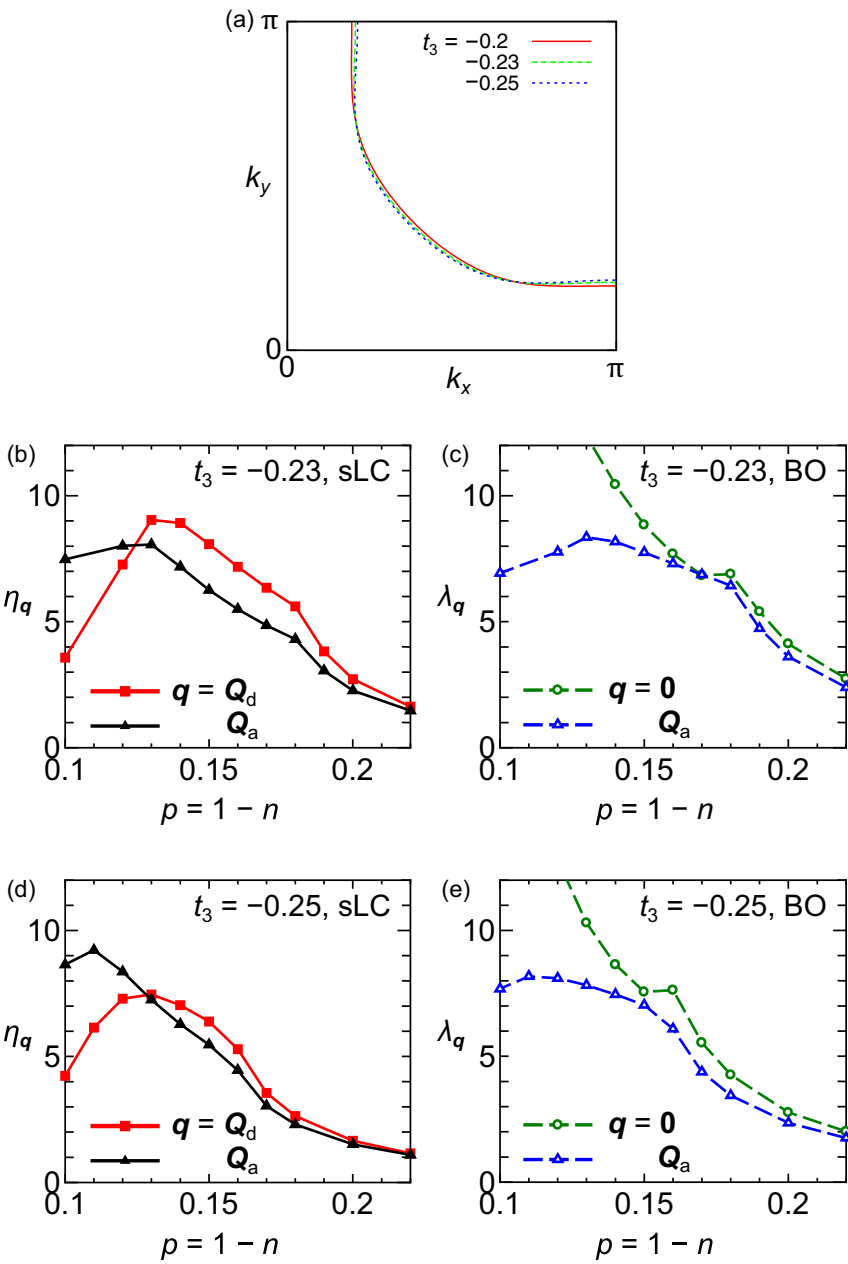

FIG. 9. (a) FSs for $t_{3}=-0.20$ to -0.25 at $n=0.85$, in the case of $t_{1}=-1.0$ and $t_{2}=1 / 6$. The set $t_{3}=0.20$ in the main text. (b)-(e) Obtained eigenvalue $\eta_{q}$ and $\lambda_{q}$ in the cases of $t_{3}=-0.23$ [(b) and (c) $]$ and $t_{3}=-0.25[(\mathrm{~d})$ and (e)].

condition $\alpha_{S}=1-0.444 p^{2}$ by following the main text.) With increasing $\left|t_{3}\right|$, the peak of sLC instability is found to shift to the underdoped region. Interestingly, the SLC eigenvalue at $\boldsymbol{q}=\boldsymbol{Q}_{\mathrm{a}}$ becomes larger than that at $\boldsymbol{q}=\boldsymbol{Q}_{\mathrm{d}}$ for $t_{3}=-0.25$. Its spin current pattern in real space is shown in Fig. 10(a).

We note that recent experiments indicate that the phase diagram of cuprate superconductors is very diverse and rich. For example, the in-plain anisotropy of the ferromagnetic susceptibility at $T=T^{*}$ is $B_{1 g}$ in YBCO [10], whereas $B_{2 g}$ in $\mathrm{Hg}$ compound [11]. Also, the antiferromagnetic susceptibility in slightly underdoped YBCO exhibits a clear symmetry breaking at a temperature between $T^{*}$ and $T_{\mathrm{CDW}}$ [58]. The present sensitive $t_{3}$ dependent SLC may give an important hint to understand diverse symmetry breaking in cuprates.

Here, we discuss the band folding and hybridization gap in the axial sLC phase. Figures 10(b) and 10(c) show the Fermi arc structures induced by the axial sLC order in the cases of (a) single- $\boldsymbol{q}$ and (b) double- $\boldsymbol{q}$ orders. We set $g^{\max } \equiv$ $\max _{\boldsymbol{k}}\left\{g_{\boldsymbol{Q}_{\mathrm{d}}}(\boldsymbol{k})\right\}=0.1$. Here, the folded band structure under the sLC order with finite $\boldsymbol{q}_{\mathrm{sLC}}$ is "unfolded" to make a comparison with ARPES results. The Fermi arc due to the single- $\boldsymbol{q}$
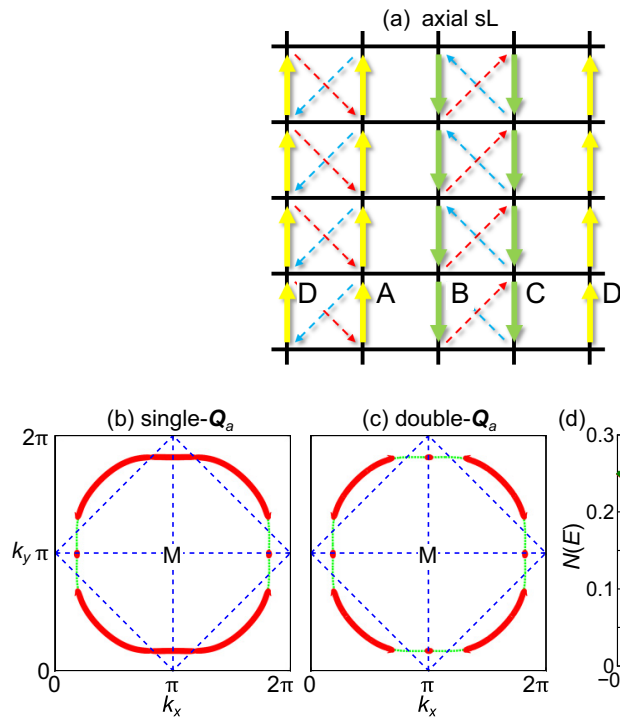

(c) double- $\boldsymbol{Q}_{a}$

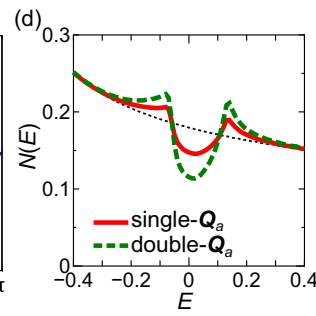

FIG. 10. (a) Schematic spin current pattern due to the sLC order at $\boldsymbol{q}=\boldsymbol{Q}_{\mathrm{a}}$. (b) Fermi arc structure due to the single- $\boldsymbol{q}$ order, (c) that due to the double- $q$ order, and (d) pseudogap in the DOS due to the axial sLC order $\left(\boldsymbol{q}=\boldsymbol{Q}_{\mathrm{a}}\right)$.

order in Fig. 10(b) belongs to the $B_{1 g}$ symmetry. The resultant pseudogap in the DOS is shown in Fig. 10(d).

\section{Reduction of eigenvalues by $z<1$}

In the present work, we study the mechanism of exotic DW orders due to the interference between paramagnons based on the linearized DW equation. The obtained form factor represents the characteristics and the symmetry of the DW, and the eigenvalue expresses the strength of the DW instability. In the present numerical study, we drop the self-energy in the DW equation. Then, the obtained eigenvalues shown in Figs. 5 and 9 are much larger than unity. In addition, the eigenvalue of the superconducting gap equation, $\lambda_{\mathrm{SC}}$, shown in Fig. $5(\mathrm{~b})$ is also very large.

The self-energy gives the quasiparticle weight as $z \equiv[1-$ $\left.\operatorname{Re} \partial \Sigma(\epsilon) /\left.\partial \epsilon\right|_{\epsilon=\mu}\right]^{-1}(<1)$, and $z^{-1}(>1)$ is the mass enhancement. The effect of the self-energy in the DW equation has been studied in Ref. [50] for Fe-based superconductors $(z \sim$ $1 / 3$ ), and it was revealed that the eigenvalue of the orbital fluctuations is reduced by the self-energy, and the orbital order temperature is reduced to $\sim 100 \mathrm{~K}$, consistent with experiments.

Here, we study the effect of the renormalization factor $z$ on the eigenvalues in the present single-orbital Hubbard model. Then, the Green function is given as

$$
G^{z}(k)=\frac{1}{i \epsilon_{n} / z-\mu-\epsilon_{k}} .
$$

First, we discuss the effect of $z$ on $\lambda_{\mathrm{SC}}$, by replacing two $G$ 's in Eq. (6) with $G^{z}$ given by Eq. (B1). On the other hand, we do not include $z$ in the susceptibilities $\chi^{s, c}$ in $V^{s, c}$ in the pairing interaction, in order not to change the Stoner factor $\alpha_{S}$. Then, it is well known that $\lambda_{\mathrm{SC}}$ is reduced as $z \lambda_{\mathrm{SC}}$ based on the Eliashberg equation [59]. 

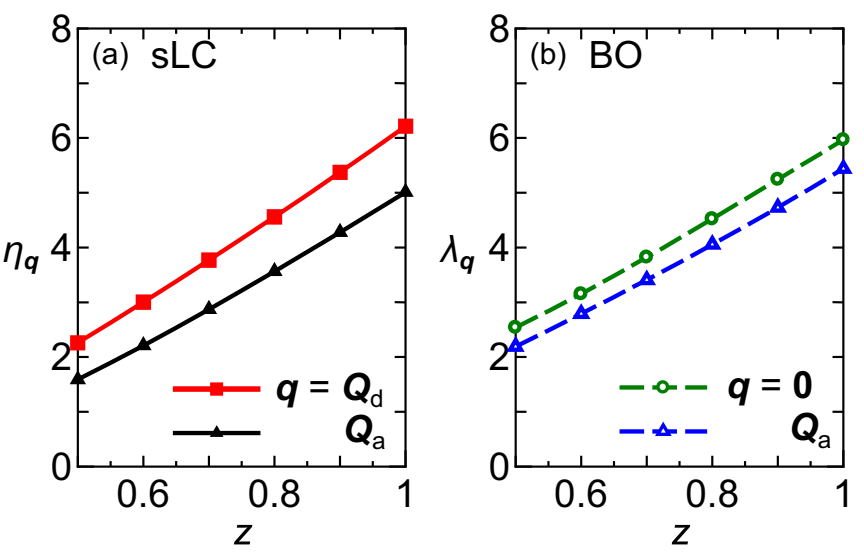

FIG. 11. Obtained eigenvalues (a) $\eta_{q}$ and (b) $\lambda_{q}$ as functions of $z$.

Next, we discuss the effect of $z$ on $\lambda_{q}\left(\eta_{q}\right)$ by replacing four $G$ 's in Eqs. (A4) and (A5) [Eqs. (A6) and (A7)] with $G^{z}$ given by Eq. (B1). The obtained $z$ dependences of the eigenvalues at $T=0.03$ are shown in Fig. 11. It is verified that both $\lambda_{q}$ and $\eta_{q}$ are reduced in proportion to $z$. Although this approximation may be justified only for $z \lesssim 1$, the obtained results strongly indicate that both $\lambda_{q}$ and $\eta_{q}$ are reduced to $O(1)$ in the case of $z \lesssim 0.2$, which is realized in cuprate superconductors.

\section{Unfolded band structure under the sLC order}

Here, we examine the experimentally observed band structure in the sLC ordered state, by applying the unfolding procedure proposed in Ref. [56]. Figure 12 shows the "unfolded" band structure in the single- $\boldsymbol{Q}_{\mathrm{d}}$ sLC order at $g^{\max }=$ 0.1 , which corresponds to Fig. 6(a) in the main text. The pseudogap closes on the $X-Y$ line owing to the odd-parity form factor. This Dirac point which will be smeared out for $T \sim T^{*}\left(\gg T_{\mathrm{CDW}}\right)$ because of very large inelastic scattering at the hot spot [5-7,40]. In addition, the Dirac point should be masked by the $d$ wave $\mathrm{BO}$ below $T_{\mathrm{CDW}}$.
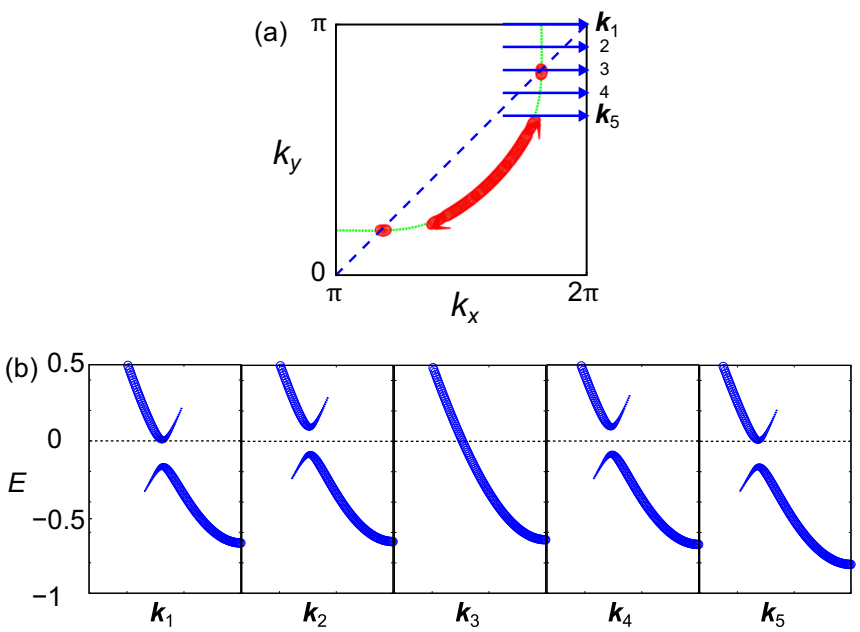

FIG. 12. The unfolded band structure in the single- $\boldsymbol{Q}_{\mathrm{d}} \mathrm{sLC}$ order corresponds to Fig. 6(a) in the main text.

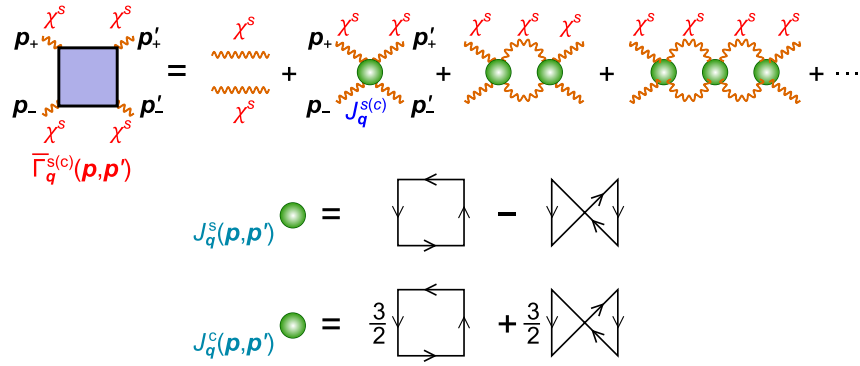

FIG. 13. Diagrammatic expression of $\bar{\Gamma}_{q}^{s(c)}\left(p, p^{\prime}\right)$, which represents the scattering process of the magnon pair through the interaction $J_{q}^{s(c)}\left(p, p^{\prime}\right)$. Mathematically, $\bar{\Gamma}_{q}^{s(c)}\left(p, p^{\prime}\right)$ diverges when magnon pairs with momentum $\boldsymbol{q}$ condense. Thus, sLC/BO is interpreted as the condensation of odd/even parity magnon pairs.

\section{APPENDIX C: BO/sLC ORDER AS MAGNON-PAIR CONDENSATION}

We explain that the sLC order is exactly the same as the magnon-pair condensation. The following spin quadrupole order occurs owing to the magnon-pair condensation [53]:

$$
K_{i, j}^{\alpha, \beta} \equiv\left\langle s_{i}^{\alpha} s_{j}^{\beta}\right\rangle-\left\langle s_{i}^{\alpha} s_{j}^{\beta}\right\rangle_{0},
$$

where $\alpha, \beta=x, y, z$, and the relation $K_{i, j}^{\alpha, \beta}=K_{j, i}^{\beta, \alpha}$ holds. We will explain that the even-parity function $a_{i, j} \equiv K_{i, j}^{\alpha, \alpha} / 3$ (with $\left.a_{i, j}=a_{j, i}\right)$ corresponds the $\mathrm{BO}$ state, and the odd-parity function $b_{i, j}^{\alpha} \equiv i \epsilon_{\alpha \beta \gamma} K_{i, j}^{\beta, \gamma} / 2$ (with $b_{i, j}^{\alpha}=-b_{j, i}^{\alpha}$ ) corresponds the sLC order.

Here, we explain that $\Gamma_{q}^{s, c}\left(k, k^{\prime}\right)$ due to the AL processes represents the scattering between two-magnons. To simplify the discussion, we drop the MT term, and consider only AL terms with two $\chi^{s}$ 's. Then, we define $\bar{\Gamma}_{q}^{s(c)}\left(p, p^{\prime}\right)$ by the following relation: $\Gamma_{q}^{c(s)}\left(k, k^{\prime}\right)=T^{2} \sum_{p, p^{\prime}}[G(k-p)+$ $(-) G(k+p)] \bar{\Gamma}_{q}^{c(s)}\left(p, p^{\prime}\right) G\left(k^{\prime}-p\right)$. Figure 13 shows the diagrammatic expression of $\bar{\Gamma}_{q}^{s, c}\left(p, p^{\prime}\right)$, which represents the scattering process of magnon pair amplitude $b^{z}(a)$ through the interaction $J_{q}^{s(c)}\left(p, p^{\prime}\right)$, which is a moderate function of $T$. With decreasing temperatures, $\bar{\Gamma}_{q}^{c(s)}\left(p, p^{\prime}\right)$ diverges when singlet (triplet) magnon pairs with momentum $\boldsymbol{q}$ condense, and the critical temperature corresponds to $\lambda_{q}=1\left(\eta_{q}=1\right)$.

Here, we introduce $\bar{f}_{\boldsymbol{q}}(k) \equiv T \sum_{p} H_{\boldsymbol{q}}(k, p) f_{\boldsymbol{q}}(p)$ and $\quad \bar{g}_{q}(k) \equiv T \sum_{p} H_{q}(k, p) g_{q}(p), \quad$ where $\quad H_{q}(k, p)=$ $G\left(p_{+}\right) G\left(p_{-}\right) G(p-k)$, and $f_{\boldsymbol{q}}(p)$ and $g_{\boldsymbol{q}}(p)$ are form factors of the DW equations. Then, the DW equations are rewritten as

$$
\begin{aligned}
& \lambda_{\boldsymbol{q}} \bar{f}_{\boldsymbol{q}}(k)=T \sum_{p} J_{\boldsymbol{q}}^{c}(k, p) \chi^{s}\left(p_{+}\right) \chi^{s}\left(p_{-}\right) \bar{f}_{\boldsymbol{q}}(p), \\
& \eta_{\boldsymbol{q}} \bar{g}_{\boldsymbol{q}}(k)=T \sum_{p} J_{\boldsymbol{q}}^{s}(k, p) \chi^{s}\left(p_{+}\right) \chi^{s}\left(p_{-}\right) \bar{g}_{\boldsymbol{q}}(k),
\end{aligned}
$$

where the kernel function $J_{q}^{c, s}(k, p)$ is given in Fig. 13. These equations means that $\bar{f}_{\boldsymbol{q}}(k)\left[\bar{g}_{\boldsymbol{q}}(k)\right]$ corresponds to the singlet (triplet) magnon pair condensation. Therefore, their Fourier transformations correspond to $a_{i, j}$ and $b_{i, j}^{z}$, respectively.

To summarize, in the present double spin-flip mechanism, magnon-pair condensation $a, b^{z} \neq 0$ occurs at $T=$ $T_{\text {sLC }}$. Therefore, the sLC/BO given by the present mecha- 


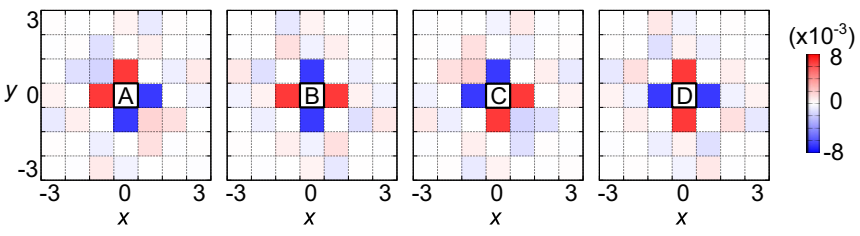

FIG. 14. Obtained spin current from the center site (A-D) to different sites under the diagonal sLC with period $4 a_{\mathrm{Cu}-\mathrm{Cu}}$. The real space pattern is depicted in Fig. 1(c), with sites A-D in a unit cell.

nism is exactly the same as "condensation of odd/even parity magnon pairs."

\section{APPENDIX D: SPONTANEOUS SPIN CURRENT IN THE SLC PHASE}

\section{Calculation of the spin current}

Here, we investigate the spin current in real space due to the "spin-dependent self-energy" $\delta t_{i, j}^{\sigma}=\sigma g_{i, j}$ shown in Fig. 3(c), which is purely imaginary and odd with respect to $i \leftrightarrow j$. The spin current operator from site $j$ to site $i$ is $j_{i, j}^{\sigma}=-i \sum_{\sigma} \sigma\left(h_{i, j}^{\sigma} c_{i \sigma}^{\dagger} c_{j \sigma}-(i \leftrightarrow j)\right)$, where $h_{i, j}^{\sigma}=t_{i, j}+$ $\delta t_{i, j}^{\sigma}$. Then, the spin current from $j$ to $i$ is given as $J_{i, j}^{s}=$ $\left\langle j_{i, j}^{S}\right\rangle_{\hat{h}^{\sigma}}$.

Here, we calculate the spin current for the commensurate sLC order at $\boldsymbol{q}_{\mathrm{sLC}}=(\pi / 2, \pi / 2)$, which is achieved by putting $n=1.0$. Then, the unit cells are composed four sites A-D. Figure 14 shows the obtained spin current $J_{i, j}^{s}$ from the center site $(j=\mathrm{A}-\mathrm{B})$ to a different site in Fig. 1(c), by setting $g^{\max }=$ 0.1 . The obtained current is $\left|J_{i, j}^{s}\right| \sim 10^{-2}$ in units $\left|t_{1}\right| / \hbar$. The derived spin current pattern between the nearest and secondnearest sites is depicted in Fig. 1(c). The spin current is exactly conserved at each site.

\section{Possible diagonal sLC patterns in real space}

Next, we explain that the spin current pattern derived from the form factor $g_{q}(\boldsymbol{k})$ in Fig. 3(b) is not uniquely determined. In fact, the form factor in real space is given as $i \operatorname{Im}\left\{g_{i, j} e^{i \psi}\right\} \sim$ $i \operatorname{Im}\left\{e^{i \boldsymbol{q} \cdot\left(\boldsymbol{r}_{i}+\boldsymbol{r}_{j}\right) / 2} e^{i \psi}\right\}$, where $\psi$ is an arbitrary phase. Here, we discuss other possible spin current patterns by choosing $\psi$.

First, we discuss the real space pattern for $\boldsymbol{q}_{\mathrm{sLC}}=$ $(\pi / 2, \pi / 2),(\pi / 2,-\pi / 2)$. We assume that Fig. 1(c) corresponds to $\psi=0$. Then, the single- $\boldsymbol{q}$ spin current pattern for $\psi=\pi / 2$ is given in Fig. 15(a). The double- $\boldsymbol{q}$ spin current order is given by the combination of the sLC order at $\boldsymbol{q}_{\mathrm{sLC}}=$ (a)

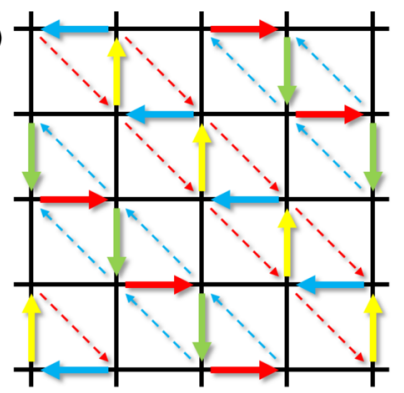

(b)

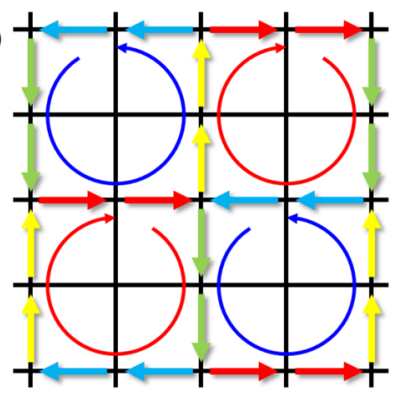

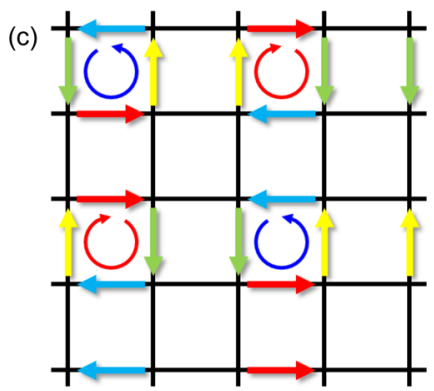

(d)

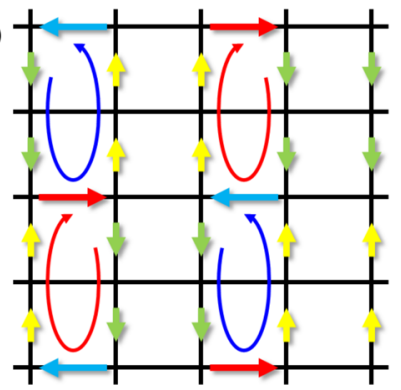

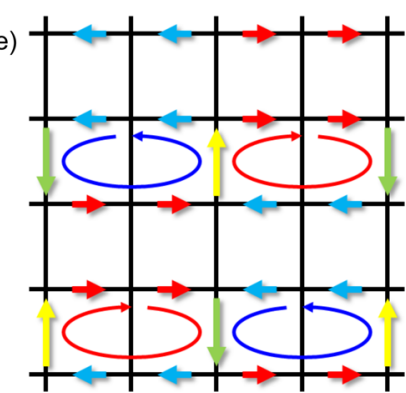

FIG. 15. Examples of the diagonal sLC pattern in real space for $\boldsymbol{q}_{\mathrm{sLC}}=(\pi / 2, \pi / 2),(\pi / 2,-\pi / 2)$. (a) Single- $\boldsymbol{q}$ sLC pattern for $\psi=$ $\pi / 2$. (b)-(e) Four examples of double- $\boldsymbol{q}$ sLC patterns.

$(\pi / 2, \pi / 2)$ and that at $\boldsymbol{q}_{\mathrm{sLC}}=(\pi / 2,-\pi / 2)$ with arbitrary phase factors. Figures 15(b) and 15(c) are given by the combination of Fig. 1(c) with its $\pi / 2$ rotation, and Figs. 15(d) and $15(\mathrm{e})$ are given by the combination of Fig. 15(a) with its $\pi / 2$ rotation. We stress that the magnitude of spin current $\left|J_{i, j}^{s}\right|$ in Figs. 15(b) and 15(c) has $C_{4}$ symmetry, whereas that in Figs. 15(d) and 15(e) breaks the $C_{4}$ symmetry.

[1] G. Ghiringhelli, M. L. Tacon, M. Minola, S. Blanco-Canosa, C. Mazzoli, N. B. Brookes, G. M. D. Luca, A. Frano, D. G. Hawthorn, F. He, T. Loew, M. M. Sala, D. C. Peets, M. Salluzzo, E. Schierle, R. Sutarto, G. A. Sawatzky, E. Weschke, B. Keimer, and $\mathrm{L}$. Braicovich, Long-range incommensurate charge fluctuations in (Y,Nd) $\mathrm{Ba}_{2} \mathrm{Cu}_{3} \mathrm{O}_{6+x}$, Science 337, 821 (2012).

[2] R. Comin, A. Frano, M. M. Yee, Y. Yoshida, H. Eisaki, E. Schierle, E. Weschke, R. Sutarto, F. He, A. Soumyanarayanan, Y. He, M. L. Tacon, I. S. Elfimov, J. E. Hffman, G. A. Sawatzky, B. Keimer, and A. Damascelli, Charge order driven by Fermiarc instability in $\mathrm{Bi}_{2} \mathrm{Sr}_{2-x} \mathrm{La}_{x} \mathrm{CuO}_{+\delta}$, Science 343, 390 (2014).
[3] Y. Kohsaka, T. Hanaguri, M. Azuma, M. Takano, J. C. Davis, and H. Takagi, Visualization of the emergence of the pseudogap state and the evolution to superconductivity in a lightly holedoped Mott insulator, Nat. Phys. 8, 534 (2012).

[4] K. Fujita, M. H. Hamidian, S. D. Edkins, C. K. Kim, Y. Kohsaka, M. Azuma, M. Takano, H. Takagi, H. Eisaki, S. Uchida, A. Allais, M. J. Lawler, E.-A. Kim, S. Sachdev, and J. C. S. Davis, Direct phase-sensitive identification of a $d$-form factor density wave in underdoped cuprates, Proc. Natl. Acad. Sci. USA 111, E3026 (2014). 
[5] B. Kyung, S. S. Kancharla, D. Senechal, A.-M. S. Tremblay, M. Civelli, and G. Kotliar, Pseudogap induced by short-range spin correlations in a doped Mott insulator, Phys. Rev. B 73, 165114 (2006).

[6] T. A. Maier, M. S. Jarrell, and D. J. Scalapino, Understanding high-temperature superconductors with quantum cluster theories, Physica C 460-462, 13 (2007).

[7] T. Moriya and K. Ueda, Spin fluctuations and high temperature superconductivity, Adv. Phys. 49, 555 (2000).

[8] A. Shekhter, B. J. Ramshaw, R. Liang, W. N. Hardy, D. A. Bonn, F. F. Balakirev, R. D. McDonald, J. B. Betts, S. C. Riggs, and A. Migliori, Bounding the pseudogap with a line of phase transitions in $\mathrm{YBa}_{2} \mathrm{Cu}_{3} \mathrm{O}_{6+\delta}$, Nature (London) 498, 75 (2013).

[9] R.-H. He, M. Hashimoto, H. Karapetyan, J. D. Koralek, J. P. Hinton, J. P. Testaud, V. Nathan, Y. Yoshida, H. Yao, K. Tanaka, W. Meevasana, R. G. Moore, D. H. Lu, S.-K. Mo, M. Ishikado, H. Eisaki, Z. Hussain, T. P. Devereaux, S. A. Kivelson, J. Orenstein, A. Kapitulnik, and Z.-X. Shen, From a single-band metal to a high-temperature superconductor via two thermal phase transitions, Science 331, 1579 (2011).

[10] Y. Sato, S. Kasahara, H. Murayama, Y. Kasahara, E.-G. Moon, T. Nishizaki, T. Loew, J. Porras, B. Keimer, T. Shibauchi, and Y. Matsuda, Thermodynamic evidence for a nematic phase transition at the onset of the pseudogap in $\mathrm{YBa}_{2} \mathrm{Cu}_{3} \mathrm{O}_{y}$, Nat. Phys. 13, 1074 (2017).

[11] H. Murayama, Y. Sato, R. Kurihara, S. Kasahara, Y. Mizukami, Y. Kasahara, H. Uchiyama, A. Yamamoto, E.-G. Moon, J. Cai, J. Freyermuth, M. Greven, T. Shibauchi, and Y. Matsuda, Diagonal nematicity in the pseudogap phase of $\mathrm{HgBa}_{2} \mathrm{CuO}_{4+\delta}$, Nat. Commun. 10, 3282 (2019).

[12] S. Nakata, M. Horio, K. Koshiishi, K. Hagiwara, C. Lin, M. Suzuki, S. Ideta, K. Tanaka, D. Song, Y. Yoshida, H. Eisaki, and A. Fujimori, Nematicity in the pseudogap state of cuprate superconductors revealed by angle-resolved photoemission spectroscopy, arXiv:1811.10028.

[13] K. Ishida, S. Hosoi, Y. Teramoto, T. Usui, Y. Mizukami, K. Itaka, Y. Matsuda, T. Watanabe, and T. Shibauchi, Divergent nematic susceptibility near the pseudogap critical point in a cuprate superconductor, J. Phys. Soc. Jpn. 89, 064707 (2020).

[14] S. Bulut, W. A. Atkinson, and A. P. Kampf, Spatially modulated electronic nematicity in the three-band model of cuprate superconductors Phys. Rev. B 88, 155132 (2013).

[15] Y. Wang and A. V. Chubukov, Charge-density-wave order with momentum $(2 Q, 0)$ and $(0,2 Q)$ within the spin-fermion model: Continuous and discrete symmetry breaking, preemptive composite order, and relation to pseudogap in hole-doped cuprates, Phys. Rev. B 90, 035149 (2014).

[16] R.-Q. Xing, L. Classen, and A. V. Chubukov, Orbital order in FeSe: The case for vertex renormalization, Phys. Rev. B 98, 041108(R) (2018).

[17] M. A. Metlitski and S. Sachdev, Instabilities near the onset of spin density wave order in metals, New J. Phys. 12, 105007 (2010); S. Sachdev and R. La Placa, Bond Order in Two-Dimensional Metals with Antiferromagnetic Exchange Interactions, Phys. Rev. Lett. 111, 027202 (2013).

[18] W. Metzner, M. Salmhofer, C. Honerkamp, V. Meden, and K. Schönhammer, Functional renormalization group approach to correlated fermion systems, Rev. Mod. Phys. 84, 299 (2012); C. Husemann and W. Metzner, Incommensurate nematic fluctuations in the two-dimensional Hubbard model, Phys. Rev. B 86,
085113 (2012); T. Holder and W. Metzner, Incommensurate nematic fluctuations in two-dimensional metals, ibid. 85, 165130 (2012); C. Honerkamp, Charge instabilities at the metamagnetic transition of itinerant electron systems, ibid. 72, 115103 (2005).

[19] J. C. S. Davis and D.-H. Lee, Concepts relating magnetic interactions, intertwined electronic orders, and strongly correlated superconductivity, Proc. Natl. Acad. Sci. USA 110, 17623 (2013).

[20] E. Berg, E. Fradkin, S. A. Kivelson, and J. M. Tranquada, Striped superconductors: how spin, charge and superconducting orders intertwine in the cuprates, New J. Phys. 11, 115004 (2009).

[21] Y. Yamakawa, and H. Kontani, Spin-Fluctuation-Driven Nematic Charge-Density Wave in Cuprate Superconductors: Impact of Aslamazov-Larkin Vertex Corrections, Phys. Rev. Lett. 114, 257001 (2015).

[22] M. Tsuchiizu, K. Kawaguchi, Y. Yamakawa, and H. Kontani, Multistage electronic nematic transitions in cuprate superconductors: A functional-renormalization-group analysis, Phys. Rev. B 97, 165131 (2018).

[23] K. Kawaguchi, Y. Yamakawa, M. Tsuchiizu, and H. Kontani, Competing unconventional charge-density-wave states in cuprate superconductors: Spin-fluctuation-driven mechanism, J. Phys. Soc. Jpn. 86, 063707 (2017).

[24] P. A. Lee, Amperean Pairing and the Pseudogap Phase of Cuprate Superconductors, Phys. Rev. X 4, 031017 (2014).

[25] D. F. Agterberg, J. C. S. Davis, S. D. Edkins, E. Fradkin, D. J. Van Harlingen, S. A. Kivelson, P. A. Lee, L. Radzihovsky, J. M. Tranquada, and Y. Wang, The physics of pair density waves, Annu. Rev. Condens. Matter Phys. 11, 231 (2020).

[26] C. M. Varma, Non-Fermi-liquid states and pairing instability of a general model of copper oxide metals, Phys. Rev. B 55, 14554 (1997).

[27] I. Affleck and J. B. Marston, Large- $n$ limit of the HeisenbergHubbard model: Implications for high- $T_{c}$ superconductors, Phys. Rev. B 37, 3774(R) (1988).

[28] F. C. Zhang, Superconducting Instability of Staggered-Flux Phase in the $t-J$ Model, Phys. Rev. Lett. 64, 974 (1990).

[29] H. J. Schulz, Fermi-surface instabilities of a generalized twodimensional Hubbard model, Phys. Rev. B 39, 2940(R) (1989).

[30] A. A. Nersesyan, G. I. Japaridze, and I. G. Kimeridze, Lowtemperature magnetic properties of a two-dimensional spin nematic state, J. Phys.: Condens. Matter 3, 3353 (1991).

[31] M. Ozaki, Broken Symmetry solutions of the extended Hubbard model, Int. J. Quantum. Chem. 42, 55 (1992).

[32] S. Zhou, K. Jiang, H. Chen, and Z. Wang, Correlation Effects and Hidden Spin-Orbit Entangled Electronic Order in Parent and Electron-Doped Iridates $\mathrm{Sr}_{2} \mathrm{IrO}_{4}$, Phys. Rev. X 7, 041018 (2017).

[33] H. Ikeda and Y. Ohashi, Theory of Unconventional Spin Density Wave: A Possible Mechanism of the Micromagnetism in Ubased Heavy Fermion Compounds, Phys. Rev. Lett. 81, 3723 (1998).

[34] S. Fujimoto, Spin Nematic State as a Candidate of the Hidden Order Phase of $\mathrm{URu}_{2} \mathrm{Si}_{2}$, Phys. Rev. Lett. 106, 196407 (2011).

[35] S. Onari and H. Kontani, Self-consistent Vertex Correction Analysis for Iron-based Superconductors: Mechanism of Coulomb Interaction-Driven Orbital Fluctuations, Phys. Rev. Lett. 109, 137001 (2012). 
[36] S. Onari, Y. Yamakawa, and H. Kontani, Sign-Reversing Orbital Polarization in the Nematic Phase of FeSe due to the $C_{2}$ Symmetry Breaking in the Self-Energy, Phys. Rev. Lett. 116, 227001 (2016).

[37] Y. Yamakawa, S. Onari, and H. Kontani, Nematicity and Magnetism in FeSe and Other Families of Fe-Based Superconductors, Phys. Rev. X 6, 021032 (2016).

[38] T. Yoshida, M. Hashimoto, I. M. Vishik, Z. X. Shen, and A. Fujimori, Pseudogap, Superconducting gap, and Fermi arc in high- $T_{c}$ cuprates revealed by angle-resolved photoemission spectroscopy, J. Phys. Soc. Jpn. 81, 011006 (2011).

[39] Y. K. Kim, O. Krupin, J. D. Denlinger, A. Bostwick, E. Rotenberg, Q. Zhao, J. F. Mitchell, J. W. Allen, and B. J. Kim, Fermi arcs in a doped pseudospin-1/2 Heisenberg antiferromagnet, Science 345, 187 (2014).

[40] H. Kontani, Anomalous transport phenomena in Fermi liquids with strong magnetic fluctuations, Rep. Prog. Phys. 71, 026501 (2008).

[41] H. Kontani, Transport Phenomena in Strongly Correlated Fermi Liquids (Springer-Verlag, Berlin, 2013).

[42] C. Stock, W. J. L. Buyers, R. Liang, D. Peets, Z. Tun, D. Bonn, W. N. Hardy, and R. J. Birgeneau, Dynamic stripes and resonance in the superconducting and normal phases of $\mathrm{YBa}_{2} \mathrm{Cu}_{3} \mathrm{O}_{6.5}$ ortho-II superconductor, Phys. Rev. B 69, 014502 (2004).

[43] R. Tazai and H. Kontani, Hexadecapole fluctuation mechanism for $s$-wave heavy fermion superconductor $\mathrm{CeCu}_{2} \mathrm{Si}_{2}$ : Interplay between intra- and inter-orbital Cooper pairs, J. Phys. Soc. Jpn. 88, 063701 (2019); R. Tazai, Y. Yamakawa, and H. Kontani, Plain $s$-wave superconductivity near the magnetic criticality: Enhancement of attractive electron-boson coupling vertex corrections, ibid. 86, 073703 (2017).

[44] R. Tazai and H. Kontani, Multipole fluctuation theory for heavy fermion systems: Application to multipole orders in $\mathrm{CeB}_{6}$, Phys. Rev. B 100, 241103(R) (2019).

[45] M. Tsuchiizu, Y. Ohno, S. Onari, and H. Kontani, Orbital Nematic Instability in the Two-Orbital Hubbard Model: Renormalization-Group + Constrained RPA Analysis, Phys. Rev. Lett. 111, 057003 (2013).

[46] R. Tazai, Y. Yamakawa, M. Tsuchiizu, and H. Kontani, Functional renormalization group study of orbital fluctuation mediated superconductivity: Impact of the electron-boson coupling vertex corrections, Phys. Rev. B 94, 115155 (2016).

[47] R. Tazai, Y. Yamakawa, M. Tsuchiizu, and H. Kontani, Prediction of $d$-wave bond-order and pseudogap in organic superconductor $\kappa$-(BEDT-TTF $)_{2} \mathrm{X}$ : Similarities to cuprate superconductors, arXiv:2010.15516.

[48] R. Tazai, Y. Yamakawa, and H. Kontani, Emergence of Charge Loop Current in Geometrically Frustrated Hubbard Model: Functional Renormalization Group Study, arXiv:2010.16109.

[49] S. Onari and H. Kontani, Origin of diverse nematic orders in Fe-based superconductors: $45^{\circ}$ rotated nematicity in $A \mathrm{Fe}_{2} \mathrm{As}_{2}$ ( $A=\mathrm{Sc}, \mathrm{Rb}$ ), Phys. Rev. B 100, 020507(R) (2019).

[50] S. Onari and H. Kontani, Hidden antiferro-nematic order in Fe-based superconductor $\mathrm{BaFe}_{2} \mathrm{As}_{2}$ and $\mathrm{NaFeAs}$ above $T_{S}$, Phys. Rev. Res. 2, 042005(R) (2020).

[51] D. J. Scalapino, E. Loh, and J. E. Hirsch, $d$-wave pairing near a spin-density-wave instability, Phys. Rev. B 34, 8190 (1986).

[52] V. Mishra and M. R. Norman, Strong coupling critique of spin fluctuation driven charge order in underdoped cuprates, Phys. Rev. B 92, 060507(R) (2015).

[53] A. F. Andreev and I. A. Grishchuk, Spin nematics, Zh. Eksp. Teor. Fiz. 87, 467 (1984).

[54] P. Chandra and P. Coleman, Quantum Spin Nematics: MomentFree Magnetism, Phys. Rev. Lett. 66, 100 (1991).

[55] N. Shannon, T. Momoi, and P. Sindzingre, Nematic Order in Square Lattice Frustrated Ferromagnets, Phys. Rev. Lett. 96, 027213 (2006).

[56] W. Ku, T. Berlijn, and C.-C. Lee, Unfolding First-Principles Band Structures, Phys. Rev. Lett. 104, 216401 (2010).

[57] K. Kawaguchi and H. Kontani, Spin-fluctuation-driven $B_{1 g}$ and $B_{2 g}$ Bond order and induced in-plane anisotropy in magnetic susceptibility in cuprate superconductors: Impact of hot-/coldspot structure on bond-order symmetry, J. Phys. Soc. Jpn. 89 , 124704 (2020).

[58] V. Hinkov, D. Haug, B. Fauque, P. Bourges, Y. Sidis, A. Ivanov, C. Bernhard, C. T. Lin, and B. Keimer, Electronic liquid crystal state in the high-temperature superconductor $\mathrm{YBa}_{2} \mathrm{Cu}_{3} \mathrm{O}_{6.45}$, Science 319, 597 (2008).

[59] P. B. Allen and B. Mitrovic, Theory of superconducting $T_{c}$, Solid State Phys. 37, 1 (1983) 